\title{
A new construction of boundary interpolating wavelets for fourth order problems
}

\author{
Silvia Bertoluzza* and Valérie Perrier ${ }^{\dagger}$
}

September 15, 2015

\begin{abstract}
In this article we introduce a new mixed Lagrange-Hermite interpolating wavelet family on the interval, to deal with two types (Dirichlet and Neumann) of boundary conditions. As this construction is a slightly modification of the interpolating wavelets on the interval of Donoho, it leads to fast decomposition, error estimates and norm equivalences. This new basis is then used in adaptive wavelet collocation schemes for the solution of one dimensional fourth order problems. Numerical tests conducted on the 1D Euler-Bernoulli beam problem, show the efficiency of the method.
\end{abstract}

\section{Introduction}

The properties of wavelet bases are nowadays well understood, and they allow the design of methods of arbitrary high order for the solution of elliptic partial differential equations, that display an optimal lowest complexity coupled with the good local behaviour necessary for local refinement [8]. Wavelet collocation methods were introduced in the mid nineties [2, 32] and have since proven their strength on second order problems arising in fluid-dynamics $[4,31,29]$ and in structural mechanics. In particular, recent works show their effectiveness in the study of buckling [20] or vibration [18] phenomena.

The wavelet collocation method is based on two (independent) ingredients: expanding the unknown solution of the equation considered in a Deslaurier-Dubuc interpolating basis [16], and determining the unknown coefficients by requiring that the equation is exactly verified at the corresponding "collocation points". The construction of interpolating wavelets on the interval by Donoho [17], allows to efficiently handle Dirichlet boundary conditions $[3,4]$. Adaptive versions of the method on non uniform grids have been also proposed, based on a quite simple adaptive strategy where the wavelet coefficients themselves are used to decide where to refine or coarsen the underlying grid.

\footnotetext{
${ }^{*}$ CNR, IMATI, Pavia, Italy (Silvia.Bertoluzza@imati.cnr.it)

†Université Grenoble-Alpes et CNRS, Laboratoire Jean Kuntzmann, F-38 000 Grenoble, France (Valerie.Perrier@imag.fr)
} 
With respect to a Galerkin approach, collocation has some advantage, in particular when non linear equations are considered [5]. Thanks to the particular structure of the Interpolating Wavelet Transform, the evaluation of local nonlinearities turns out to be quite easy, also in an adaptive framework, and collocation allows to avoid the need of computing integrals involving non linear expressions, which, in the wavelet context, can be quite cumbersome.

In this article we aim at extending such a method to the numerical solution of fourth order problems. Such problems occur in a variety of applications including structural mechanics (beams, plates, ...), fluid dynamics (ice formation, fluids in the lungs), nanotechnologies, or image denoising. We should point out that, in this context, Galerkin methods using orthogonal wavelet bases have been already successfully employed in previous works $[22$, $23,15]$. The first problem that we need to face is the handling of the boundary conditions. Focusing, for the moment, on the one dimensional case, we will have to impose two boundary conditions at each of the two extrema of the interval. In order to maintain the balance between the number of equations and the number of degrees of freedom, we will then need to enrich the space with one more function per extremum. To this aim, we introduce a new family of interpolating wavelets on the interval, that we will call Mixed Lagrange-Hermite interpolatory wavelet bases. It consists in adding a boundary interpolating function at each extremum, satisfying an Hermite interpolation property (vanishing at all nodes and with first derivative equals to one at one node) and in modifying the other (Lagrange) interpolating boundary functions, in such a way that their derivative vanishes at the extrema. This new interpolating wavelet family will be used to develop our adaptive collocation schemes for the solution of one-dimensional fourth order problems.

The rest of the paper is organized as follows: Section 2 introduces the construction of the boundary mixed Lagrange-Hermite interpolating wavelets, and states the main properties satisfied by this new wavelet basis. Section 3 describes their use in the framework of an adaptive collocation wavelet schemes, and presents first numerical tests in 1D on two EulerBernulli beam problems.

\section{Interpolating boundary Hermite wavelets on the In- terval}

To construct our new boundary modified interpolating wavelet basis on the interval, we start with an Interpolating Multiresolution Analysis (MRA) on the line [17, 24]. Let $\phi$ be a Deslaurier-Dubuc scaling function on $\mathbb{R}$, which, we recall, satisfies an interpolation property: $\phi(0)=1$ and $\phi(k) \neq 0, \forall k \neq 0$. Such function $\phi$ can be constructed by autocorrelation from a Daubechies orthonormal compactly scaling function $\Phi$ with $N+1$ vanishing moments [14]:

$$
\phi(x)=\Phi * \Phi(-\cdot)(x) .
$$

We let $L=2 N+1$ denote the polynomial reproduction allowed by $\phi$, i.e., for each polynomial $p$ of degree lower or equal than $L$ we have the identity $p(x)=\sum_{k} p(k) \phi(x-k)$. Moreover, we let $R>1$ be the regularity of $\phi$, such that $\phi$ is Hölder continuous of order $R$, which, for 

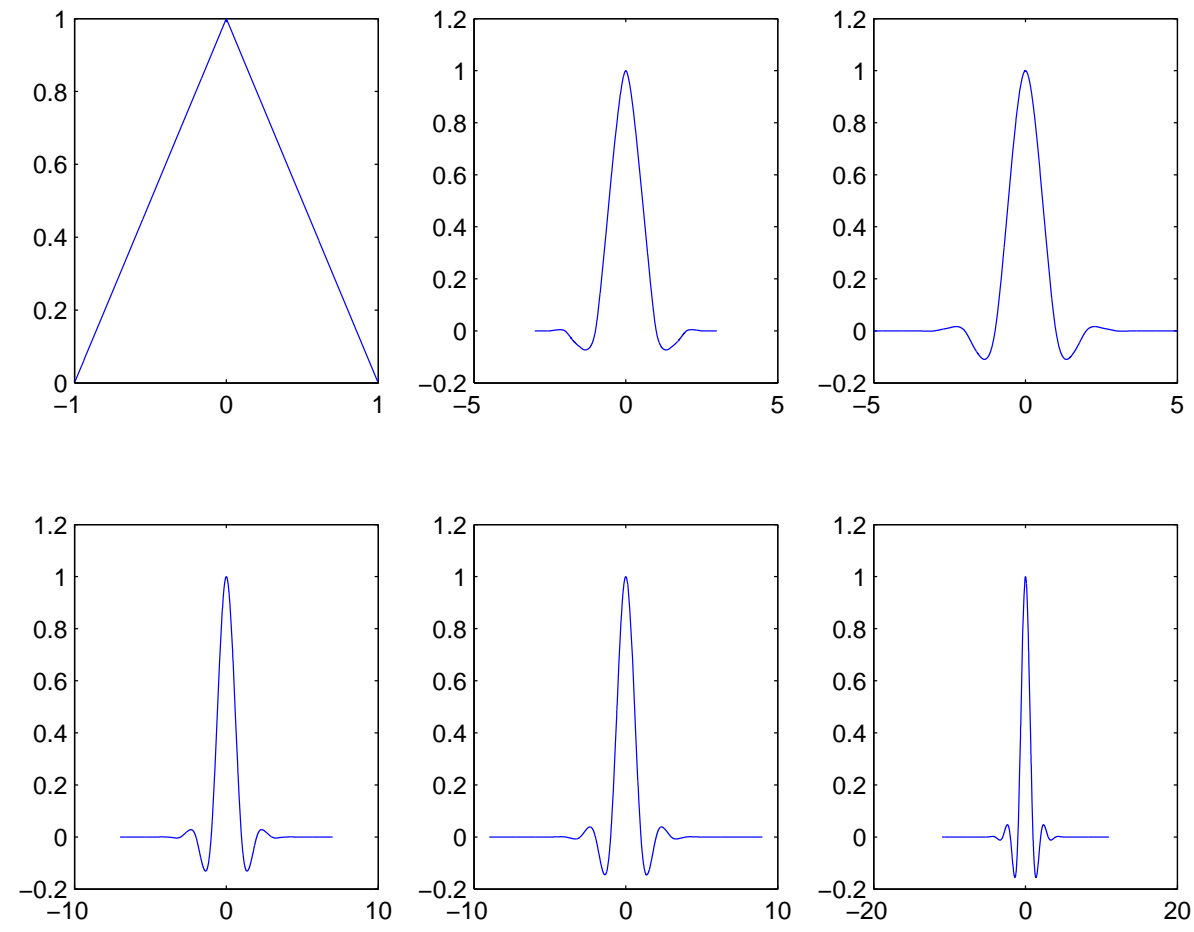

Figure 1: Deslaurier-Dubuc functions $\phi$ for $N=0, \cdots, 5$

simplicity, we will assume to be integer. We observe that $\operatorname{supp} \phi=[-L, L]$. Figure 1 shows different functions $\phi$ obtained from different Daubechies scaling functions.

We will construct in the following a MRA on the interval $(0,1)$, with interpolating functions at interior points and allowing an Hermite interpolation at boundaries 0 and 1 . This MRA will be a slight modification of interpolating MRA on the interval introduced by [17], obtained by adding at each scale one function at each boundary, allowing the interpolation of the derivative at 0 and 1 , while modifying the other boundary functions in such a way that their derivative vanishes at 0 and 1 . We remark that this MRA does not fall in the framework of Hermite Multiwavelets constructed in [12].

We aim at constructing our MRA on $(0,1)$ in such a way that any continuously differentiable function can be expanded as a linear combination of interpolating functions as follows:

$$
f=\sum_{k=-1}^{2^{j_{0}+1}} \beta_{j_{0}, k} \varphi_{j_{0}, k}+\sum_{j \geq j_{0}} \sum_{k=0}^{2^{j}} \alpha_{j, k} \psi_{j, k}
$$

where $j_{0}$ will be specified in the following, and where the functions $\varphi_{j_{0}, k}$ and $\psi_{j, k}$ satisfy a mixed Lagrange-Hermite interpolating condition:

$$
\begin{array}{cll}
\varphi_{j_{0}, k}\left(k^{\prime} 2^{-j}\right)=2^{j_{0} / 2} \delta_{k, k^{\prime}}, \forall k^{\prime}=0, \cdots, 2^{j_{0}} & \text { and } & \varphi_{j_{0}, k}^{\prime}(0)=\varphi_{j_{0}, k}^{\prime}(1)=0, \\
\phi_{j_{0},-1}\left(k^{\prime} 2^{-j}\right)=0, \forall k^{\prime}=0, \cdots, 2^{j_{0}} & \text { and } \quad\left(\phi_{j_{0},-1}\right)^{\prime}(0)=1, \quad\left(\phi_{j_{0},-1}\right)^{\prime}(0)=0, \\
\phi_{j_{0}, 2^{j}+1}\left(k^{\prime} 2^{-j}\right)=0, \forall k^{\prime}=0, \cdots, 2^{j_{0}} & \text { and } \quad\left(\phi_{j_{0}, 2^{j_{0}+1}}\right)^{\prime}(1)=0, \quad\left(\phi_{j_{0}, 2^{j_{0}+1}}\right)^{\prime}(1)=0, \\
\psi_{j, k}\left(k^{\prime} 2^{-j-1}\right)=2^{3 j / 2} \delta_{2 k+1, k^{\prime}}, \quad \forall j \geq j_{0} & \text { and } \quad \forall k^{\prime}=0, \cdots, 2^{j+1} .
\end{array}
$$

The convergence of the series (2) in Sobolev spaces will be a consequence of Theorem 2.2. 


\subsection{Construction of the basis}

The construction follows the same approach as in [17]. Let $j_{0}=\left\lceil\log _{2}(L-1)\right\rceil$, and let $j \geq j_{0}$. Let $f \in C^{0}([0,1])$ be continuously differentiable at 0 and 1 . We construct a sequence $\left\{\tilde{\beta}_{j, k} ; k \in \mathbb{Z}\right\}$ in such a way that

$$
\begin{gathered}
\sum_{k \in \mathbb{Z}} \tilde{\beta}_{j, k} \phi_{j, k}\left(n / 2^{j}\right)=f\left(n / 2^{j}\right), n=0, \cdots, 2^{j}, \\
\sum_{k \in \mathbb{Z}} \tilde{\beta}_{j, k} \phi_{j, k}^{\prime}(0)=f^{\prime}\left(0^{+}\right), \quad \sum_{k \in \mathbb{Z}} \tilde{\beta}_{j, k} \phi_{j, k}^{\prime}(1)=f^{\prime}\left(1^{-}\right),
\end{gathered}
$$

where, throughout this section we set, as usual, $\phi_{j, k}(x)=2^{j / 2} \phi\left(2^{j} x-k\right)$. To do so, we let $\left\{\beta_{j, k}=2^{-j / 2} f\left(k 2^{-j}\right) ; k=0, \ldots 2^{j}\right\}$ be normalized samples of $f$ in $[0,1]$, and $\left\{\beta_{j}^{h b}=\right.$ $\left.2^{-3 j / 2} f^{\prime}\left(0^{+}\right), \beta_{j}^{h \sharp}=2^{-3 j / 2} f^{\prime}\left(1^{-}\right)\right\}$its normalized derivative values (resp. right derivative and left derivative) at boundaries 0 and 1 . The extrapolated samples $\left\{\tilde{\beta}_{j, k} ; k<0\right\}$ are defined by introducing the Lagrange-Hermite polynomial $\mathcal{H}_{j, L}^{b}$ of degree $L$ interpolating $f$ at the $L$ leftmost samples, as well as its derivative at 0 :

$$
\mathcal{H}_{j, L}^{b}\left(k 2^{-j}\right)=f\left(k 2^{-j}\right) \text { for } 0 \leq k \leq L-1 \text { and }\left(\mathcal{H}_{j, L}^{b}\right)^{\prime}(0)=f^{\prime}\left(0^{+}\right)
$$

and using this to extrapolate to $k<0$ :

$$
\tilde{\beta}_{j, k}=2^{-j / 2} \mathcal{H}_{j, L}^{b}\left(k 2^{-j}\right), \quad k<0 .
$$

Similarly the samples $\left\{\tilde{\beta}_{j, k} ; k>2^{j}\right\}$ are obtained by introducing the Lagrange-Hermite polynomial $\mathcal{H}_{j, L}^{\sharp}$ of degree $L$ interpolating $f$ at the $L$ rightmost samples, as well as its derivative at 1 :

$$
\mathcal{H}_{j, L}^{\sharp}\left(k 2^{-j}\right)=f\left(k 2^{-j}\right) \text { for } 2^{j}-L+1 \leq k \leq 2^{j} \text { and }\left(\mathcal{H}_{j, L}^{\sharp}\right)^{\prime}(1)=f^{\prime}\left(1^{-}\right) .
$$

Using this to extrapolate to $k>2^{j}$ :

$$
\tilde{\beta}_{j, k}=2^{-j / 2} \mathcal{H}_{j, L}^{\sharp}\left(k 2^{-j}\right), \quad k>2^{j},
$$

the extrapolation operator $\mathcal{E}_{j, L}$ of degree $L$ at level $j$ is then defined by:

$$
\mathcal{E}_{j, L}\left(\left(\beta_{j, k}\right)_{k=0}^{2^{j}}, \beta_{j}^{h b}, \beta_{j}^{h \sharp}\right)=\left(\tilde{\beta}_{j, k}\right)_{j \in \mathbb{Z}},
$$

where $\tilde{\beta}_{j, k}$ coincides with $\beta_{j, k}$ for $k=0, \cdots, 2^{j}$, and is defined by (3) for $k<0$ and (4) for $k>2^{j}$.

Definition 2.1. Let $V_{j}$ be the collection of the restrictions to $[0,1]$ of all functions $\tilde{f}$ defined by:

$$
\tilde{f}=\sum_{k \in \mathbb{Z}} \tilde{\beta}_{j, k} \phi_{j, k}
$$

where the coefficients $\tilde{\beta}_{j, k}$ are the extrapolated samples, defined by relation (5), of the normalized node values of some function $f$. 
The following properties hold.

Property 2.1. The space $V_{j}$ is a mixed Lagrange-Hermite interpolating space in the sense that for each function $\tilde{f} \in V_{j}$, defined by (6) with coefficients obtained by extrapolation of the normalized samples of a function $f$, it holds that $\tilde{f}\left(k 2^{-j}\right)=2^{j / 2} \beta_{j, k}=f\left(k 2^{-j}\right) \quad \forall k=0, \cdots 2^{j}$, and $\tilde{f}^{\prime}(0)=f^{\prime}\left(0^{+}\right), \tilde{f}^{\prime}(1)=f^{\prime}\left(1^{-}\right)$.

Proof. $\tilde{f}\left(k 2^{-j}\right)=2^{j / 2} \tilde{\beta}_{j, k}=2^{j / 2} \beta_{j, k}$ for $k=0, \cdots 2^{j}$ due to the interpolation property of scaling functions: $\phi_{j, k}\left(n 2^{-j}\right)=2^{j / 2} \delta_{k, n}$.

The property on the derivatives follows from the fact that $\tilde{f}$ is a $C^{1}$-function ( $\phi$ is assumed to be $C^{R}$ with $R>1$ ), and polynomial outside $[0,1]$, and has for right-derivative $f^{\prime}\left(0^{+}\right)$at 0 and left-derivative $f^{\prime}\left(1^{-}\right)$at 1 . By $C^{1}$-continuity, one gets: $\tilde{f}^{\prime}(0)=f^{\prime}\left(0^{+}\right), \tilde{f}^{\prime}(1)=f^{\prime}\left(1^{-}\right)$.

Together with the definition of $V_{j}$, property 2.1 gives us a condition which is necessary and sufficient for a function $f$ to belong to $V_{j}: f \in C^{1}([0,1])$ belongs to $V_{j}$ if and only if

$$
f=\left.\sum_{k} \tilde{\beta}_{j, k} \phi_{j, k}\right|_{[0,1]},
$$

where $\tilde{\beta}_{j, k}$ are the extrapolated samples, defined by relation (5), of the normalized node values of $f$ itself.

Property 2.2. $V_{j}$ reproduces the polynomials of degree $\leq L$ : if $\Pi_{L}$ denotes the space of polynomials of degree less than $L$ on $[0,1]$, one has $\Pi_{L} \subset V_{j}$.

Proof. Property 2.2 follows from the unicity of interpolating polynomial, and the reproduction of polynomials allowed by the $\phi_{j, k}$ on $\mathbb{R}[17]$.

We now exhibit the basis functions of the space $V_{j}$. We first define the left extrapolation weights: $\forall n \in\{1, \cdots, L-1\}$ and $\forall k \in \mathbb{Z}$,

$$
a_{k, n}^{b}=L_{j, n}^{b}\left(k 2^{-j}\right), \quad a_{k, 0}^{b}=H_{j, 0}^{b}\left(k 2^{-j}\right), \quad b_{k}^{b}=2^{j} H_{j, 1}^{b}\left(k 2^{-j}\right),
$$

where the Lagrange-Hermite polynomials $L_{j, n}^{b}, H_{j, 0}^{b}, H_{j, 1}^{b}$ are the interpolation polynomials needed to define $\mathcal{H}_{j, L}^{b}$, that are defined respectively as follows:

- for $n=1, \cdots, L-1, L_{j, n}^{b}$ is the polynomial assuming the value 1 in $n / 2^{j}$, vanishing at $k / 2^{j}$ for $k=0, \cdots, L-1, k \neq n$ and whose derivative vanishes in 0 ,

- $H_{j, 0}^{b}$ is the polynomial vanishing at $k / 2^{j}, k=1, \cdots, L-1$, assuming value 1 at 0 and whose derivative vanishes in 0 ,

- $H_{j, 1}^{b}$ is the polynomial vanishing at $k / 2^{j}, k=0, \cdots, L-1$, and whose derivative assumes the value 1 in 0 . 

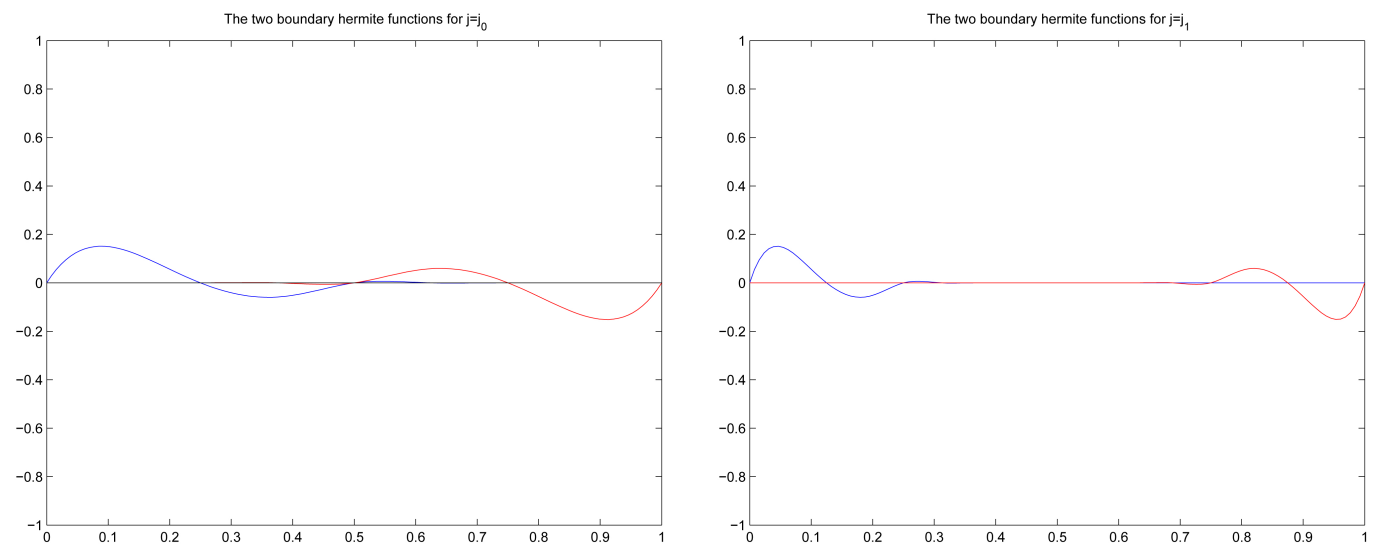

Figure 2: The Hermite functions $\varphi_{j}^{h b}$ (in blue) and $\varphi_{j}^{h \sharp}$ (in red), for $j=2$ (left) and $j=3$ (right)

The exact analytic expression for such polynomials are given in Annexe 4 (see equations $(34,35,36))$. Remark that the weights are indeed independent of $j$. With such weights we have

$$
\tilde{\beta}_{j, k}=\beta_{j}^{h b} b_{k}^{b}+\sum_{n=0}^{L-1} a_{k, n}^{b} \beta_{j, n} \quad \forall k<0 .
$$

For $j \geq j_{1}=\left\lfloor\log _{2}(L-1)\right\rfloor+2$, the left boundary scaling functions are then defined by:

$$
\begin{aligned}
\varphi_{j, n}^{b} & =\phi_{j, n}+\sum_{k=-L+1}^{-1} a_{k, n}^{b} \phi_{j, k}, \quad 0 \leq n \leq L-1 \\
\varphi_{j}^{h b} & =\phi_{j, 0}+\sum_{k=-L+1}^{-1} b_{k}^{b} \phi_{j, k}
\end{aligned}
$$

Remark that equations $(7,8)$ also make sense for $j=0$, so that they allow us to define functions $\phi_{0, n}^{b}, n=0, \cdots, L-1$, and $\phi_{0}^{h b}$, such that using the the scaling invariance of the $\phi_{j, n}$, we obtain $\varphi_{j, n}^{b}(x)=2^{j / 2} \varphi_{0, n}^{b}\left(2^{j} x\right)$ and $\varphi_{j, n}^{h b}(x)=2^{j / 2} \varphi_{0, n}^{h b}\left(2^{j} x\right)$.

Similarly, the right extrapolation weights are defined by: $\forall n \in\{1, \cdots, L-1\}$ and $\forall k \in \mathbb{Z}$,

$$
a_{k, n}^{\sharp}=L_{j, n}^{\sharp}\left(\left(2^{j}-k\right) 2^{-j}\right), \quad a_{k, 0}^{\sharp}=H_{j, 0}^{\sharp}\left(\left(2^{j}-k\right) 2^{-j}\right), \quad b_{k}^{\sharp}=2^{j} H_{j, 1}^{\sharp}\left(\left(2^{j}-k\right) 2^{-j}\right)
$$

where the Lagrange-Hermite polynomials $L_{j, n}^{\sharp}, H_{j, 0}^{\sharp}, H_{j, 1}^{\sharp}$ are defined analogously to $L_{j, n}^{b}, H_{j, 0}^{b}$ and $L_{j, 1}^{b}$ and are used to build $\mathcal{H}_{j, L}^{\sharp}$ (see once again Annexe 4). Then

$$
\tilde{\beta}_{j, 2^{j}-k}=\beta_{j}^{h \sharp} b_{k}^{\sharp}+\sum_{n=0}^{L-1} a_{k, n}^{\sharp} \beta_{j, 2^{j}-n} \quad \forall k<0
$$


The right boundary scaling functions are then defined by:

$$
\begin{aligned}
\varphi_{j, 2^{j}-n}^{\sharp} & =\phi_{j, 2^{j}-n}+\sum_{k=-L+1}^{-1} a_{k, n}^{\sharp} \phi_{j, 2^{j}-k}, 0 \leq n \leq L-1 \\
\varphi_{j}^{h \sharp} & =\phi_{j, 2^{j}}+\sum_{k=-L+1}^{-1} b_{k}^{\sharp} \phi_{j, 2^{j}-k}
\end{aligned}
$$

These boundary scaling functions are completed by the "interior" scaling functions $\varphi_{j, k}=$ $2^{j / 2} \phi\left(2^{j} x-k\right), k=L, \cdots, 2^{j}-L$ to span the space $V_{j}$, and the following proposition holds.

Proposition 2.1. For $j \geq j_{1}, V_{j}$ is a finite dimensional space of dimension $2^{j}+3$ and it satisfies:

$$
V_{j}=\operatorname{span}\left\{\varphi_{j, k}^{b}\right\}_{k=0}^{L-1} \oplus \operatorname{span}\left\{\varphi_{j}^{h b}\right\} \oplus \operatorname{span}\left\{\varphi_{j, k}\right\}_{k=L}^{2^{j}-L} \oplus \operatorname{span}\left\{\varphi_{j, k}^{\sharp}\right\}_{k=2^{j}-L+1}^{2^{j}} \oplus \operatorname{span}\left\{\varphi_{j}^{h \sharp}\right\}
$$

Moreover, every $f \in V_{j}$ has the representation:

$$
\tilde{f}=\sum_{k=0}^{L-1} \beta_{j, k} \varphi_{j, k}^{b}+\beta_{j}^{h b} \varphi_{j}^{h b}+\sum_{k=L}^{2^{j}-L} \beta_{j, k} \varphi_{j, k}+\sum_{k=2^{j}-L+1}^{2^{j}} \beta_{j, k} \varphi_{j, k}^{\sharp}+\beta^{h \sharp} \varphi_{j}^{h \sharp}
$$

with the coefficients: $\beta_{j, k}=2^{-j / 2} f\left(k 2^{-j}\right), \beta_{j}^{h b}=2^{-3 j / 2} f^{\prime}\left(0^{+}\right), \beta_{j}^{h \sharp}=2^{-3 j / 2} f^{\prime}\left(1^{-}\right)$.

Remark 2.1. For $j \geq j_{1}$ we have that $2^{-j}(L-1)<1-2^{-j}(L-1)$ and then the samples used in the left hand side extrapolation $(k<0)$ are disjoint from the samples used in the right hand side extrapolation $\left(k>2^{j}\right)$. For $j \in\left[j_{0}, j_{1}-1\right]$ the left and the right hand side extrapolations share some of the nodes. As a result we obtain some basis functions which have contributions both from the left and the right side of the interval. More precisely, for $2^{j}-L+1 \leq n \leq L-1$ we can introduce the function

$$
\varphi_{j, n}^{b \sharp}=\phi_{j, n}+\sum_{k=-L+1}^{-1} a_{k, n}^{b} \phi_{j, k}+\sum_{k=-L+1}^{-1} a_{k, 2^{j}-n}^{\sharp} \phi_{j, 2^{j}-k} .
$$

We then have the representation, for $j_{0} \leq j<j_{1}$

$$
V_{j}=\operatorname{span}\left\{\varphi_{j, k}^{b}\right\}_{k=0}^{2^{j}-L} \oplus \operatorname{span}\left\{\varphi_{j}^{h b}\right\} \oplus \operatorname{span}\left\{\varphi_{j, k}^{b \sharp}\right\}_{k=2^{j}-L+1}^{L-1} \oplus \operatorname{span}\left\{\varphi_{j, k}^{\sharp}\right\}_{k=L}^{2^{j}} \oplus \operatorname{span}\left\{\varphi_{j}^{h \sharp}\right\} .
$$

We have the following Lemma.

Lemma 2.1. The sequence $\left\{V_{j}\right\}_{j \geq j_{0}}$ is nested:

$$
V_{j} \subset V_{j+1}
$$

Proof. We start by recalling a fundamental property of the Deslaurier-Dubuc MRA [17]. Let

$$
U_{j}=\operatorname{span}\left\{\phi_{j, k} ; \quad k \in \mathbb{Z}\right\} \in L_{\text {loc }}^{2}(\mathbb{R}) .
$$


Let $f=\sum_{k \in \mathbb{Z}} 2^{-j / 2} f\left(k 2^{-j}\right) \phi_{j k} \in U_{j}$. Then the values of $f$ at dyadic points at level $j+1$ can be obtained from the values at the dyadic points at level $j$ by centered polynomial interpolation of order $L=2 N+1$. More precisely we have that

$$
f\left((2 k+1) 2^{-(j+1)}\right)=\pi_{k}^{j}\left((2 k+1) 2^{-(j+1)}\right)
$$

with $\pi_{k}^{j} \in \Pi_{L}$ defined by

$$
\pi_{k}^{j}\left(n 2^{-j}\right)=f\left(n 2^{-j}\right), \quad n=k-N, \cdots, k+N+1 .
$$

We next recall that $V_{j}$ can be characterized as the restriction to $[0,1]$ of the subspace of $U_{j}$ (which by abuse of notation we will also denote by $V_{j}$ ) whose coefficients are obtained through the extrapolation procedure $\mathcal{E}_{j, L}$ defined in (5): $f \in V_{j}$ if and only if $f=\sum_{k} \beta_{j k} \phi_{j k}$ with

$$
\left(2^{j / 2} \beta_{j k}\right)_{k}=\mathcal{E}_{j, L}\left(f(0), f\left(2^{-j}\right), \cdots, f\left(1-2^{-j}\right), f(1), 2^{-j} f^{\prime}(0), 2^{-j} f^{\prime}(1)\right) .
$$

Let then $f \in V_{j}$ be the restriction to $[0,1]$ of $f=\sum_{k} \beta_{j k} \phi_{j k} \in U_{j}$ satisfying (14). Since $U_{j} \subseteq U_{j+1}$ we have that $f \in U_{j+1}$ and $f=\sum_{k} \beta_{j+1, k} \phi_{j+1, k}$ with coefficients $\beta_{j+1, k}=$ $2^{-(j+1) / 2} f\left(k 2^{-(j+1)}\right)$, given by (12-13). In order to prove that $f \in V_{j+1}$ it is then sufficient to prove that $\left(\beta_{j+1, k}\right)_{k}=\left(\tilde{\beta}_{j+1, k}\right)_{k}$ with

$$
\left(2^{\frac{j+1}{2}} \tilde{\beta}_{j+1, k}\right)_{k}:=\mathcal{E}_{j+1, L}\left(f(0), f\left(2^{-(j+1)}\right), \cdots, f\left(1-2^{-(j+1)}\right), f(1), 2^{-(j+1)} f^{\prime}(0), 2^{-(j+1)} f^{\prime}(1)\right) .
$$

By the definition of $\mathcal{E}_{j+1, L}$, for $k=0, \cdots, 2^{j+1}$ we have that $\tilde{\beta}_{j+1, k}=2^{-(j+1) / 2} f\left(k / 2^{j+1}\right)=$ $\beta_{j+1, k}$. We then need to prove the identity for $k<0$ and $k>2^{j+1}$. To fix the ideas let us consider the $k<0$ case. Let $p=\mathcal{H}_{j, L}^{b}$ denote the polynomial of degree $L=2 N+1$ that verifies

$$
p\left(k 2^{-j}\right)=f\left(k 2^{-j}\right), \quad k=0, \cdots, L-1, \quad p^{\prime}(0)=f^{\prime}(0),
$$

(used in the extrapolation $\left.\mathcal{E}_{j, L}\right)$. Since $f \in V_{j}$ we have that $f\left(k / 2^{j}\right)=p\left(k / 2^{j}\right)$ for $k \leq L-1$. Using (12) it is then immediate to verify that $f\left(k / 2^{j+1}\right)=p\left(k / 2^{j+1}\right)$, for $k \leq L-1$ : for even values of $k$, this is trivial, while for odd values of $k=2 n+1$ we have

$$
f\left(k / 2^{j+1}\right)=\pi_{k}^{j}\left(k / 2^{j+1}\right)=p\left(k / 2^{j+1}\right),
$$

since $\pi_{k}^{j}$ coincides with $f$ (and therefore with $\left.p\right)$ at the $L+1$ nodes $(k-N) / 2^{j}, \cdots,(k+N+$ 1) $/ 2^{j}$. Letting now $q=\mathcal{H}_{j+1, L}^{b}$ denote the polynomial of degree $L$ that verifies

$$
q\left(k 2^{-(j+1)}\right)=f\left(k 2^{-(j+1)}\right), \quad k=0, \cdots, L-1, \quad q^{\prime}(0)=f^{\prime}(0),
$$

it is immediate to check that $q=p$ (since $q\left(k / 2^{j+1}\right)=p\left(k / 2^{j+1}\right), k=0, \cdots, L-1$ and $\left.q^{\prime}(0)=p^{\prime}(0)\right)$. It is now easy to conclude that for $k<0$

$$
\tilde{\beta}_{j+1, k}=2^{-(j+1)} q\left(2^{-(j+1)} k\right)=2^{-(j+1)} p\left(2^{-(j+1)} k\right)=2^{-(j+1)} f\left(2^{-(j+1)} k\right)=\beta_{j+1, k} .
$$

An analogous argument yields $\tilde{\beta}_{j+1, k}=\beta_{j+1, k}$ for $k>2^{j+1}$. 

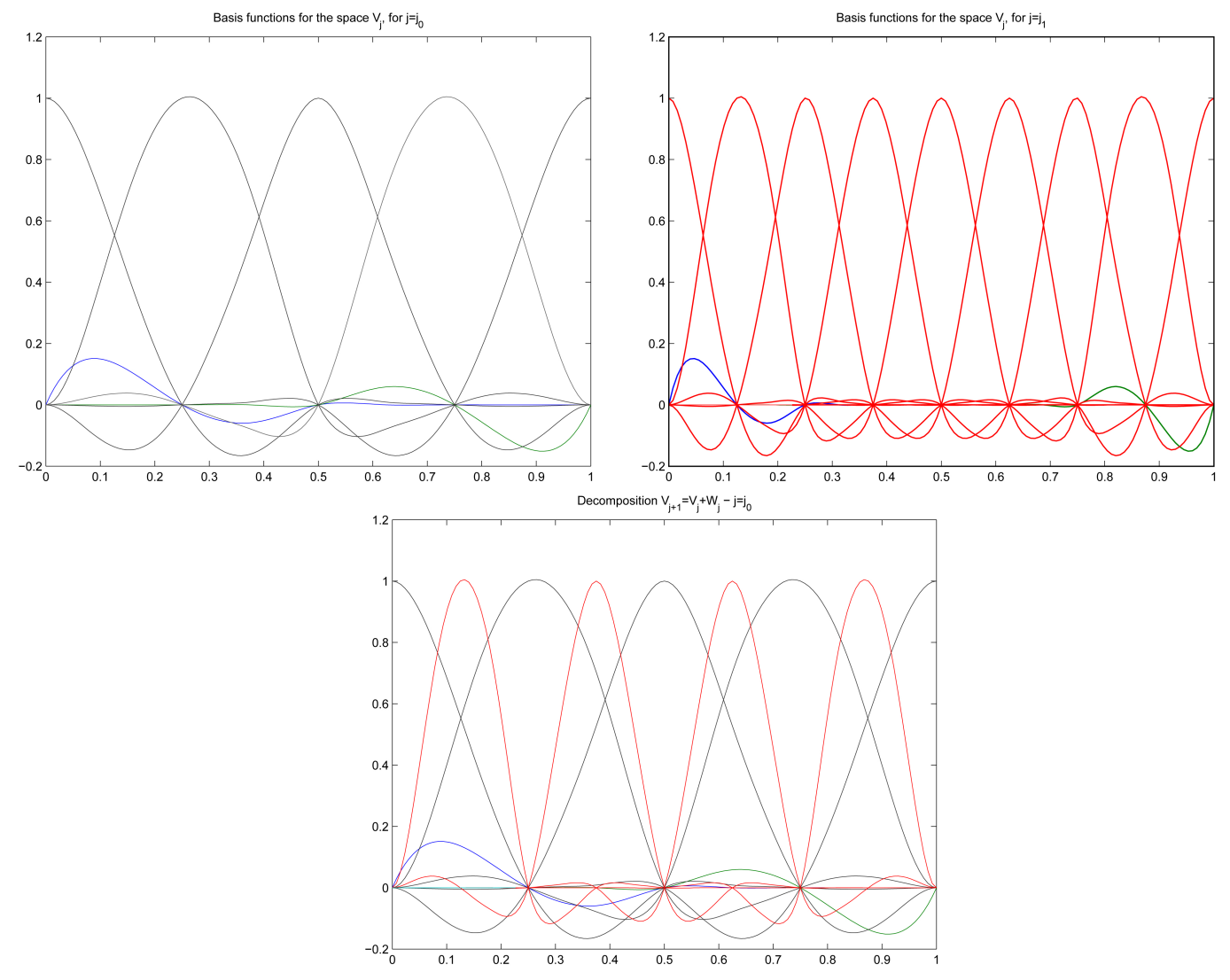

Figure 3: The basis functions for the spaces $V_{j_{0}}$ (top-left) and $V_{j_{1}}$ (top-right), and the multiscale basis for $V_{j_{1}}$ obtained from the splitting of the form (20) (bottom). 


\subsection{Interpolation and wavelets}

From now on, in order to simplify the notation, we will denote the boundary scaling functions of $V_{j}$ by $\varphi_{j, k}:=\varphi_{j, k}^{b}$ for $k=0, \ldots, L-1$ and $\varphi_{j, k}:=\varphi_{j, k}^{\sharp}$ for $k=2^{j}-L+1, \ldots, 2^{j}$. The "Hermite" scaling functions $\varphi_{j}^{h b}$ and $\varphi_{j}^{h \sharp}$ will be respectively denoted by $\varphi_{j,-1}$ and $\varphi_{j, 2^{j}+1}$. This leads to the uniform representation:

$$
V_{j}=\operatorname{span}\left\{\varphi_{j, k} ; k=-1, \ldots, 2^{j}+1\right\}
$$

where, of course, the scaling functions $\varphi_{j, k}$ for $k<L$ and $k>2^{j}-L$ do not coincide those on $\mathbb{R}: \varphi_{j, k}(x) \neq \phi_{j, k}(x)=2^{j / 2} \phi\left(2^{j} x-k\right)$.

We may then define the interpolation operator $\mathcal{I}_{j}: C^{1}([0,1]) \rightarrow V_{j}$

$$
\mathcal{I}_{j} f=2^{-3 j / 2} f^{\prime}(0) \varphi_{j,-1}+2^{-j / 2} \sum_{k=0}^{2^{j}} f\left(k 2^{-j}\right) \varphi_{j k}+2^{-3 j / 2} f^{\prime}(1) \varphi_{j, 2^{j}+1} .
$$

Thanks to the polynomial reproduction property the following error estimate holds.

Theorem 2.2. If $f \in H^{s}(0,1)$ with $3 / 2<s<L+1,0 \leq r \leq\lceil R\rceil-1, r \leq s$ then

$$
\left\|f-\mathcal{I}_{j} f\right\|_{H^{r}(0,1)} \lesssim 2^{-j(s-r)}|f|_{H^{s}(0,1)} .
$$

Proof. We prove the result for $j \geq j_{1}$ (the proof for $j<j_{1}$ being analogous). Let $r$ be an integer. We have

$$
\left|f-\mathcal{I}_{j} f \|_{H^{r}(0,1)}^{2}=\sum_{k=0}^{2^{j}-1}\right| f-\left.\mathcal{I}_{j} f\right|_{H^{r}\left(K_{j, k}\right)} ^{2}, \quad \text { with } K_{j, k}=\left(k / 2^{j},(k+1) / 2^{j}\right) .
$$

Now, for $p \in \Pi_{L}$ arbitrary we can write:

$$
\left|f-\mathcal{I}_{j} f\right|_{H^{r}\left(K_{j, k}\right)}=\left|(f+p)-I_{j}(f+p)\right|_{H^{r}\left(K_{j, k}\right)} \leq|f+p|_{H^{r}\left(K_{j, k}\right)}+\left|\mathcal{I}_{j}(f+p)\right|_{H^{r}\left(K_{j, k}\right)} .
$$

We distinguish three cases: $k \leq L-1, L \leq k \leq 2^{j}-L$, and $k \geq 2^{j}-L+1$. Let us consider the first case. For $k \leq L-1$ the modified right boundary functions $\varphi_{j}^{h \sharp}$ and $\varphi_{j, 2^{j}-n}^{\sharp}$, $0 \leq n \leq L-1$, vanish on $K_{j, k}$ and then, for $x \in K_{j, k}$, we have

$$
\begin{aligned}
\mathcal{I}_{j} g(x)=\sum_{n=-1}^{2^{j+1}} \beta_{j, n} \varphi_{j, n}(x)= & \sum_{n=-1}^{k+L-1} \beta_{j, n} \varphi_{j, n}(x)=\beta_{j,-1} 2^{j / 2} \varphi_{0}^{h b}\left(2^{j} x\right) \\
& +\sum_{n=0}^{L-1} \beta_{j, n} 2^{j / 2} \varphi_{0, n}^{b}\left(2^{j} x\right)+\sum_{n=L}^{k+L-1} \beta_{j, n} 2^{j / 2} \phi\left(2^{j} x-n\right),
\end{aligned}
$$

with

$$
\beta_{j,-1}=2^{-3 j / 2} g^{\prime}(0), \quad \beta_{j, n}=2^{-j / 2} g\left(n / 2^{j}\right), n \geq 0 .
$$


For $g=f+p$ we let $\hat{g}$ be such that

$$
g(x)=2^{j / 2} \hat{g}\left(2^{j} x\right)
$$

and we set

$$
\hat{h}(x)=\beta_{j,-1} \varphi_{0}^{h b}(x)+\sum_{n=0}^{L-1} \beta_{j, n} \varphi_{0, n}^{b}(x)+\sum_{n=L}^{k+L-1} \beta_{j, n} \phi(x-n),
$$

so that $\mathcal{I}_{j} g(x)=2^{j / 2} \hat{h}\left(2^{j} x\right)$. We have

$$
\left|g-\mathcal{I}_{j} g\right|_{H^{r}\left(K_{j, k}\right)}=2^{j r}|\hat{g}-\hat{h}|_{H^{r}\left(K_{0, k}\right)} \leq 2^{j r}\left(|\hat{g}|_{H^{r}\left(K_{0, k}\right)}+|\hat{h}|_{H^{r}\left(K_{0, k}\right)}\right) .
$$

Let us consider the contribution of $\hat{h}$. We easily see that

$$
\begin{aligned}
|\hat{h}|_{H^{r}\left(K_{0, k}\right)} & \lesssim \max \left\{\left|\beta_{j, n}\right|, n=-1, \cdots, k+L-1\right\} \\
& \lesssim\|\hat{g}\|_{W^{1, \infty}([0,2 L-2])} \lesssim\|\hat{g}\|_{H^{s}([0,2 L-2])},
\end{aligned}
$$

yielding

$$
\left|g-\mathcal{I}_{j} g\right|_{H^{r}\left(K_{j, k}\right)} \lesssim 2^{j r}\|\hat{g}\|_{H^{s}([0,2 L-2])} .
$$

Letting $\hat{f}$ and $\hat{p}$ be such that $f(x)=2^{j / 2} \hat{f}(2 x)$ and $p(x)=2^{j / 2} \hat{p}\left(2^{j} x\right)$, so that $\hat{g}=\hat{f}+\hat{p}$, and given the arbitrariness of $p$ we immediately obtain

$$
\left|f-\mathcal{I}_{j} f\right|_{H^{r}\left(K_{j, k}\right)} \lesssim 2^{j r} \inf _{\hat{p} \in \Pi_{L}}\|\hat{f}+\hat{p}\|_{H^{s}((0,2 L-2))} \lesssim 2^{j r}|\hat{f}|_{H^{s}(0,2 L-2)},
$$

where the last inequality is obtained by applying Theorem 3.1.1 in [7]. A change of variable finally yields

$$
\left|f-\mathcal{I}_{j} f\right|_{H^{r}\left(K_{j, k}\right)} \lesssim 2^{j(r-s)}|f|_{H^{s}\left(0,2^{-j}(2 L-2)\right)} .
$$

Analogously, for $L \leq k \leq 2^{j}-L$ we prove

$$
\left|f-\mathcal{I}_{j} f\right|_{H^{r}\left(K_{j, k}\right)} \lesssim 2^{j(r-s)}|f|_{H^{s}\left(2^{-j}(k-L+1), 2^{-j}(k+L-1)\right)},
$$

and for $k \geq 2^{j}-L+1$ we prove

$$
\left|f-\mathcal{I}_{j} f\right|_{H^{r}\left(K_{j, k}\right)} \lesssim 2^{j(r-s)}|f|_{H^{s}\left(2^{-j}\left(2^{j}-2 L+2\right), 1\right)} .
$$

Squaring and adding up we obtain

$$
\left|f-\mathcal{I}_{j} f\right|_{\left.\left.H^{r}(] 0,1\right]\right)} \lesssim 2^{j(r-s)}|f|_{H^{s}(] 0,1[)} .
$$

which easily yields the thesis.

In addition to the direct type inequality given by Theorem 2.2, the space $V_{j}$ inherits from $U_{j}$ an inverse type inequality.

Proposition 2.3. For all $0 \leq r \leq s<R$, for all $f \in V_{j}$ it holds that

$$
\|f\|_{\left.H^{s}(00,1]\right)} \lesssim 2^{j(r-s)}\|f\|_{H^{r}(00,1]} .
$$


Let us now introduce the wavelet space $W_{j} \subset V_{j+1}$ defined as

$$
W_{j}=\left(\mathcal{I}_{j+1}-\mathcal{I}_{j}\right) V_{j}=\operatorname{span}\left\{\varphi_{j+1,2 k+1} ; k=0, \ldots, 2^{j}-1\right\} .
$$

For analogy with orthonormal wavelets we introduce the notation

$$
\psi_{j k}=\varphi_{j+1,2 k+1} .
$$

We may remark that the function $\psi_{j k}$ verifies

$$
\psi_{j k}\left((2 n+1) / 2^{j+1}\right)=2^{\frac{j+1}{2}} \delta_{k n}, \quad \text { and } \quad \psi_{j k}\left(n / 2^{j^{\prime}}\right)=0, \forall j^{\prime} \leq j, n=0, \cdots, 2^{j^{\prime}}
$$

It is easy to check that

$$
V_{j+1}=V_{j} \oplus W_{j}
$$

Such kind of multiresolution analysis has been extensively investigated in the Lagrangeinterpolating case [17]. In the present case, it is possible to define a Lagrange-Hermite interpolating wavelet transform mapping any continuously differentiable function into the sequence of its coefficients

$$
f \rightarrow\left\{\left\{\beta_{j_{0}, k}, k=0, \cdots, 2^{j_{0}}\right\},\left\{\alpha_{j_{0}, k}, k=1, \cdots, 2^{j_{0}}\right\},\left\{\alpha_{j_{0}+1, k}, k=1, \cdots, 2^{j_{0}+1}\right\}, \ldots\right\} .
$$

The following proposition, which can be proven analogously to the corresponding proposition in [17], holds.

Proposition 2.1. (IWT) Any continuously differentiable function $f \in C^{1}([0,1])$ may be reconstructed from its transform by means of

$$
f=\sum_{k=-1}^{2^{j_{0}+1}} \beta_{j_{0}, k} \varphi_{j_{0}, k}+\sum_{j \geq j_{0}} \sum_{k=0}^{2^{j}} \alpha_{j, k} \psi_{j, k}=\mathcal{I}_{j_{0}} f+\sum_{j \geq j_{0}} \mathcal{Q}_{j} f,
$$

the operator $\mathcal{I}_{j} f$ being defined in (15), and with $\mathcal{Q}_{j}=\mathcal{I}_{j+1}-\mathcal{I}_{j}$, with uniform convergence of partial sums (with respect to $j$ ). The scaling wavelet coefficients $\beta_{j_{0}, k}$ correspond to:

$$
\begin{aligned}
\beta_{j_{0}, k} & =2^{-j_{0} / 2} f\left(k 2^{-j_{0}}\right), \quad \forall k=0, \ldots 2^{j} \\
\beta_{j_{0},-1} & =2^{-3 j_{0} / 2} f^{\prime}(0) \quad \text { and } \beta_{j, 2_{0}^{j}+1}=2^{-3 j_{0} / 2} f^{\prime}(1)
\end{aligned}
$$

whereas the wavelet coefficients $\alpha_{j, k}$ are given by:

$$
\alpha_{j, k}=2^{-j / 2}\left[f\left((k+1 / 2) 2^{-j}\right)-\mathcal{I}_{j} f\left((k+1 / 2) 2^{-j}\right)\right] .
$$




\subsection{Norme equivalences}

One of the relevant properties of wavelet bases is the possibility of expressing equivalent norms for a wide range of functional spaces in terms of wavelet coefficients. This also holds true for the new wavelet basis we just introduced, provided, of course, the functional space considered is continuously embedded in $C^{1}$. In view of the application to the solution of PDEs, we concentrate here on Sobolev spaces. The following theorem holds.

Theorem 2.4. Let $f \in C^{1}([0,1])$. Then $f \in H^{s}(] 0,1[), 3 / 2<s<R$ if and only if

$$
\|f\|_{s}^{2}:=\sum_{k=-1}^{2^{j_{0}+1}}\left|\beta_{j_{0}}\right|^{2}+\sum_{j \geq 0} \sum_{k=1}^{2^{j}} 2^{2 j s}\left|\alpha_{j k}\right|^{2}<+\infty .
$$

Moreover $\|\cdot\|_{s}$ is an equivalent norm for $H^{s}(] 0,1[)$.

Proof. For $j \geq j_{0}$ let $\mathcal{Q}_{j}=\mathcal{I}_{j+1}-\mathcal{I}_{j}$. We start by proving that if $\|f\|_{s}<+\infty$ then $f \in H^{s}(] 0,1[)$. In fact we have

$$
\|f\|_{\left.\left.H^{s}(] 0,1\right]\right)}^{2}=\left\|\mathcal{I}_{j_{0}} f+\sum_{j \geq j_{0}} \mathcal{Q}_{j} f\right\|_{\left.\left.H^{s}(] 0,1\right]\right)}^{2} \lesssim\left\|\mathcal{I}_{j_{0}} f\right\|_{H^{s}(] 0,1[)}^{2}+\left\|\sum_{j \geq j_{0}} \mathcal{Q}_{j} f\right\|_{\left.\left.H^{s}(] 0,1\right]\right)}^{2}
$$

Now we have

$$
\left\|\sum_{j \geq j_{0}} \mathcal{Q}_{j} f\right\|_{\left.\left.H^{s}(] 0,1\right]\right)}^{2}=\sum_{j \geq j_{0}}\left\|\mathcal{Q}_{j} f\right\|_{\left.\left.H^{s}(] 0,1\right]\right)}^{2}+2 \sum_{j \geq j_{0}} \sum_{k>j}\left\langle\mathcal{Q}_{j} f, \mathcal{Q}_{k} f\right\rangle_{\left.H^{s}(] 0,1\right]} .
$$

We recall that the following bound holds for all $f \in H^{s}(] 0,1[), g \in H^{s+2 \varepsilon}(] 0,1[)$, provided $\varepsilon>0$ is sufficiently small:

$$
\langle f, g\rangle_{\left.H^{s}(j 0,1]\right)} \lesssim\|f\|_{H^{s-2 \varepsilon}(j 0,1)}\|g\|_{\left.\left.H^{s+2 \varepsilon}(] 0,1\right]\right)}
$$

Then we can write

$$
\begin{aligned}
\sum_{j} \sum_{k>j}\left\langle\mathcal{Q}_{j} f, \mathcal{Q}_{k} f\right\rangle_{\left.H^{s}(0,1]\right)} & \lesssim \sum_{j} \sum_{k>j}\left\|\mathcal{Q}_{j} f\right\|_{\left.\left.H^{s+2 \varepsilon}(] 0,1\right]\right)}\left\|\mathcal{Q}_{k} f\right\|_{H^{s-2 \varepsilon}(] 0,1[)} \\
& \lesssim \sum_{j} 2^{j s}\left\|\mathcal{Q}_{j} f\right\|_{\left.\left.L^{2}(] 0,1\right]\right)} \sum_{k>j} 2^{-2 \varepsilon|j-k|} 2^{k s}\left\|\mathcal{Q}_{k} f\right\|_{L^{2}([0,1])} \\
& \lesssim \sqrt{\sum_{j} 2^{2 j s}\left\|\mathcal{Q}_{j} f\right\|_{\left.\left.L^{2}(] 0,1\right]\right)}^{2}} \sqrt{\sum_{j}\left|b_{j}\right|^{2}}
\end{aligned}
$$

with

$$
b_{j}=\sum_{k>j} 2^{-2 \varepsilon|j-k|} 2^{k s}\left\|\mathcal{Q}_{k} f\right\|_{L^{2}(] 0,1[)} .
$$

Using the $\ell^{2}$ boundedness of the convolution product with $\ell^{1}$ convolution kernel we have

$$
\left\|\left(b_{j}\right)_{j}\right\|_{\ell^{2}}^{2} \lesssim \sum_{j \geq j_{0}} 2^{2 j s}\left\|\mathcal{Q}_{j} f\right\|_{\left.L^{2}(] 0,1\right]}^{2} .
$$


Using (22) and (24) we obtain

$$
\left\|\sum_{j \geq j_{0}} \mathcal{Q}_{j} f\right\|_{H^{s}(] 0,1[)}^{2} \lesssim \sum_{j \geq j_{0}} 2^{2 j s}\left\|\mathcal{Q}_{j} f\right\|_{L^{2}(] 0,1[)}^{2} .
$$

In order to prove the converse inequality we consider a decomposition $f=\bar{f}_{m_{0}}+$ $\sum_{m \geq m_{0}} \bar{d}_{m}$ of the function $f \in H^{s}(] 0,1[)$ in a Daubechies orthonormal wavelet basis with a sufficiently high number of zero moments, in such a way that for all $r, 0 \leq r \leq L+1$ the following norm equivalence holds:

$$
\|f\|_{H^{r}(] 0,1[)}^{2} \simeq\left\|\bar{f}_{m_{0}}\right\|_{L^{2}(] 0,1[)}^{2}+\sum_{m \geq m_{0}} 2^{2 m r}\left\|\bar{d}_{m}\right\|_{L^{2}(] 0,1[)}^{2} .
$$

We can then bound $\left\|\left(\mathbf{1}-\mathcal{I}_{j}\right) f\right\|_{L^{2}(] 0,1[)}$ as follows

$$
\left\|\left(\mathbf{1}-\mathcal{I}_{j}\right) f\right\|_{L^{2}(] 0,1[)} \leq\left\|\left(\mathbf{1}-\mathcal{I}_{j}\right) \bar{f}_{m_{0}}\right\|_{L^{2}(] 0,1[)}+\sum_{m \geq m_{0}}\left\|\left(\mathbf{1}-\mathcal{I}_{j}\right) \bar{d}_{m}\right\|_{L^{2}(] 0,1[)}
$$

Let us bound the different terms on the right hand side. Let $\sigma<L+1-s$. Using Theorem 2.2 , we have

$$
\left\|\left(\mathbf{1}-\mathcal{I}_{j}\right) \bar{f}_{m_{0}}\right\|_{L^{2}(] 0,1[)} \lesssim 2^{-j(s+\sigma)}\left\|\bar{f}_{m_{0}}\right\|_{H^{s+\sigma}(] 0,1[)} \lesssim 2^{-j(s+\sigma)}\left\|\bar{f}_{m_{0}}\right\|_{L^{2}(] 0,1[)} .
$$

Let us now bound the term $\left\|\left(\mathbf{1}-\mathcal{I}_{j}\right) \bar{d}_{m}\right\|_{L^{2}(] 0,1[)}$. Let us at first consider the case $m \leq j$. We have

$$
\left\|\left(\mathbf{1}-\mathcal{I}_{j}\right) \bar{d}_{m}\right\|_{L^{2}(] 0,1[)} \lesssim 2^{-j(s+\sigma)}\left\|\bar{d}_{m}\right\|_{H^{s+\sigma}(] 0,1[)} \lesssim 2^{(m-j) s} 2^{-\sigma|m-j|}\left\|\bar{d}_{m}\right\|_{L^{2}(] 0,1[)} .
$$

For $m \geq j$ we have

$$
\left\|\left(\mathbf{1}-\mathcal{I}_{j}\right) \bar{d}_{m}\right\|_{L^{2}(] 0,1[)} \lesssim 2^{-j(s-\sigma)}\left\|\bar{d}_{m}\right\|_{H^{s-\sigma}(] 0,1[)} \lesssim 2^{(m-j) s} 2^{-\sigma|m-j|}\left\|\bar{d}_{m}\right\|_{L^{2}(] 0,1[)} .
$$

Since $\mathcal{Q}_{j} f=\left(\mathbf{1}-\mathcal{I}_{j}\right) f-\left(\mathbf{1}-\mathcal{I}_{j+1}\right) f$, it is not difficult to verify that

$$
2^{s j}\left\|\mathcal{Q}_{j} f\right\|_{L^{2}(] 0,1[)} \lesssim 2^{-j \sigma}\left\|\bar{f}_{m_{0}}\right\|_{L^{2}(] 0,1[)}+\sum_{m} 2^{-\sigma|m-j|} 2^{m s}\left\|\bar{d}_{m}\right\|_{L^{2}(] 0,1[)}
$$

Using once again the boundedness of the convolution operator we can show that

$$
\sum_{j} 2^{2 j s}\left\|\mathcal{Q}_{j} f\right\|_{L^{2}(] 0,1[)}^{2} \lesssim\left\|\bar{f}_{m_{0}}\right\|_{L^{2}(] 0,1[)}^{2}+\sum_{m} 2^{2 m s}\left\|\bar{d}_{m}\right\|_{L^{2}(] 0,1[)}^{2} \lesssim\|f\|_{H^{s}(] 0,1[)}^{2}
$$

With a similar argument we obtain

$$
\left\|\mathcal{I}_{j_{0}} f\right\|_{L^{2}(] 0,1[)}^{2} \lesssim\|f\|_{L^{2}(] 0,1[)}^{2}+\left\|\left(1-\mathcal{I}_{j_{0}}\right) f\right\|_{L^{2}(] 0,1[)}^{2} \lesssim\|f\|_{H^{s}(] 0,1[)}^{2} .
$$

Finally we have proved that:

$$
\|f\|_{H^{s}(] 0,1[)}^{2} \simeq\left\|\mathcal{I}_{j_{0}} f\right\|_{L^{2}(] 0,1[)}^{2}+\sum_{j \geq j_{0}}\left\|\mathcal{Q}_{j} f\right\|_{L^{2}(] 0,1[)}^{2} .
$$


We conclude by observing that

$$
\left\|\mathcal{I}_{j_{0}} f\right\|_{L^{2}(] 0,1[)}^{2} \simeq \sum_{k=-1}^{2^{j_{0}}+1}\left|\beta_{j_{0} k}\right|^{2}
$$

(since the space $V_{j_{0}}$ is finite dimensional and all norms are equivalent), and that

$$
\left\|\mathcal{Q}_{j} f\right\|_{L^{2}(] 0,1[)}^{2} \simeq \sum_{k=0}^{2^{j}}\left|\alpha_{j k}\right|^{2},
$$

which can be obtained by using Lemma 7.6 and Lemma 7.7 of [17].

\section{$3 \quad$ Numerical solution of Fourth order problems}

We want now to demonstrate the potential of the new basis for the solution of first order problems. In order to do so, in the following, we will show on classical test-cases, how our construction of mixed Lagrange-Hermite basis functions used within a collocation scheme allows to define an accurate numerical scheme, provided a multiple precision is used in selected computations.

\subsection{Collocation wavelet method for fourth order problem}

Let us consider a one-dimensional fourth-order boundary value problem of the form: Find $u:[0,1] \rightarrow \mathbb{R}$ such that

$$
\mathcal{A} u=f \text { in }] 0,1\left[, \quad \mathcal{B}^{b} u(0)=g^{b}, \quad \mathcal{B}^{\sharp} u(1)=g^{\sharp}\right.
$$

where $\mathcal{A}$ is a fourth order operator, and $\mathcal{B}^{b}=\left(\mathcal{B}_{1}^{b}, \mathcal{B}_{2}^{b}\right): C^{1}([0,1]) \rightarrow \mathbb{R}^{2}, \mathcal{B}^{\sharp}=\left(\mathcal{B}_{1}^{\sharp}, \mathcal{B}_{2}^{\sharp}\right)$ : $C^{1}([0,1]) \rightarrow \mathbb{R}^{2}$ denotes the boundary operators on the left and right boundary respectively.

In this section we adapt to the case of fourth order problems the classical wavelet collocation method introduced in [2] for second order problems, by considering the modified interpolated wavelets introduced in section 2, and the corresponding multiresolution analysis spaces $\left(V_{J}\right)$. For a fixed $J>0$, we introduce the grid $\mathcal{G}_{J}$ of dyadic points at resolution $2^{J}$ in $[0,1]$ :

$$
\mathcal{G}_{J}=\left\{k / 2^{J}: k=0, \cdots, 2^{J}\right\}
$$

We observe that each interior point $k / 2^{J} \in \mathcal{G}_{J}, k=1, \cdots, 2^{J}-1$ corresponds to the basis function $\varphi_{J k}$, while each of the two extrema $0=0 / 2^{J}$ and $1=2^{J} / 2^{J}$ corresponds to two basis functions, namely for $x=0$ we have the two basis functions $\varphi_{J,-1}$ and $\varphi_{J, 0}$ while for $x=1$ we have the two functions $\varphi_{J, 2^{J}}$ and $\varphi_{J, 2^{J}+1}$. The collocation formulation of Problem (26) writes:

find $u_{J} \in V_{J}$ such that

$$
\begin{gathered}
\mathcal{A} u_{J}\left(k 2^{-J}\right)=f\left(k 2^{-J}\right) \quad k=1, \cdots, 2^{J}-1, \\
\mathcal{B}^{b} u_{J}(0)=g^{b}, \mathcal{B}^{\sharp} u_{J}(1)=g^{\sharp} .
\end{gathered}
$$


The unknown $u_{J}=\sum_{k=-1}^{2^{J}+1} u_{J k} \varphi_{J k}$ is sought by solving the linear system

$$
A_{J} \bar{u}_{J}=\bar{f}_{J}
$$

where the matrix $A_{J}$ is defined as follows

$$
\begin{gathered}
A_{n+2, k+2}=\mathcal{A} \varphi_{J k}\left(n 2^{-J}\right), \quad n=1, \cdots, 2^{J}-1, k=-1, \cdots, 2^{J}+1 \\
A_{i, k+2}=\mathcal{B}_{i}^{b} \varphi_{J k}(0), \quad A_{2^{J}+i+1, k+2}=\mathcal{B}_{i}^{\sharp} \varphi_{J k}(1), \quad i=1,2, \quad k=-1, \cdots, 2^{J}+1,
\end{gathered}
$$

and where the vectors $\bar{u}_{J}$ and $\bar{f}_{J}$ are, respectively, the vector of the coefficients of the unknown function $u_{J}$ and the vector of the values of the function $f$ at the interior dyadic points and of the functions $g^{b}$ at 0 and $g^{\sharp}$ at 1 .

\subsubsection{Collocation on non-uniform grids}

It has been shown, in the case of second order problems, that the wavelet collocation method is well suited to be used in the framework of adaptivity. By using the wavelet coefficients as a guide to decide whether to refine or coarsen the grid, it is possible to design non uniform grids and corresponding non uniform wavelet spaces, well suited to the approximation of a given problem. This holds true also in the case of fourth order problem. In order to define the solution of a fourth order problem on a given non uniform grid (to be selected by a suitable adaptive refinement procedure), it will be convenient to introduce the following notation for dyadic points corresponding to the scaling functions $\varphi_{j k}$ and $\psi_{j k}$

$$
x_{j k}=k 2^{-j}, k=0, \cdots, 2^{j}, \quad y_{j k}=(2 k-1) 2^{-(j+1)}, k=1, \cdots, 2^{j} .
$$

We observe that the grid $\mathcal{G}_{J}$ can be obtained as

$$
\mathcal{G}_{J}=\mathcal{G}_{j_{0}} \cup_{j=j_{0}}^{J-1}\left\{y_{j k}, k=1, \cdots, 2^{j}\right\}=\mathcal{G}_{j_{0}} \cup\left\{y_{\lambda}, \lambda \in \Lambda\right\},
$$

with $\Lambda=\left\{(j, k), j \geq j_{0}, k=1, \cdots, 2^{j}\right\}$. If we let $\Lambda_{h} \subset \Lambda$ be any finite set of $\Lambda$ we can consider the grid

$$
\mathcal{G}_{h}=\mathcal{G}_{j_{0}} \cup\left\{y_{\lambda}, \lambda \in \Lambda_{h}\right\}
$$

and the corresponding finite dimensional space

$$
\mathcal{V}_{h}=V_{j_{0}} \oplus \operatorname{span}\left\{\psi_{\lambda}, \lambda \in \Lambda_{h}\right\}
$$

We then consider the problem: find $u_{h} \in \mathcal{V}_{h}$ such that

$$
\mathcal{A} u_{h}(\lambda)=f(\lambda) \forall \lambda \in \mathcal{G}_{h} \backslash\{0,1\}, \quad \mathcal{B}^{b} u_{h}(0)=g^{b}, \mathcal{B}^{\sharp} u_{h}(1)=g^{\sharp} .
$$

\subsection{A simple adaptive strategy}

The simplest way to construct a non-uniform approximation space well suited to the solution of a given problem is to use the simple adaptive strategy already in use for second order problem [3, 4], which is essentially the one initially proposed in [27]. The idea, heuristically justified by the norm equivalence (21), is to use the absolute value of the coefficients 
themselves as indication of the possible lack of regularity (and therefore need of employing a finer grid). A very small coefficient tells us that the corresponding point is not relevant, that is the solution is already approximated at a coarser grid. The point can then be removed without deteriorating the quality of the approximation. A big coefficient, on the other hand, indicates that either the function or one of its derivatives has a sharp variation in a neighborhood of the corresponding point, and that a locally finer grid might be necessary to achieve a good approximation. The adaptive strategy is then defined as follows. We choose two (small) tolerance parameters $\varepsilon_{R}$ and $\varepsilon_{C}$ with $0<\varepsilon_{C}<\varepsilon_{R}$, and we let $\mathcal{G}_{h}$ and $\mathcal{V}_{h}$ and $u_{h}=u_{h}^{0}+\sum_{\lambda \in \Lambda_{h}} u_{\lambda} \psi_{\lambda}$ denote the actual grid and corresponding space and the actual solution (satisfying (29)). The next grid is defined according to the following strategy. For each $\lambda \in \Lambda_{h}$ three things can occur:

1. $\left|u_{\lambda}\right| \leq \varepsilon_{C}$

2. $\varepsilon_{C}<\left|u_{\lambda}\right|<\varepsilon_{R}$;

3. $\left|u_{\lambda}\right| \geq \varepsilon_{R}$.

In the case 1 . we remove the point $y_{\lambda}$ from the grid. In the case 2 . we leave $y_{\lambda}$ in the grid as it is. In the third case we leave $y_{\lambda}$ in the new grid, and we add two neighbouring points at the next level. More precisely, if for $\lambda=(j, k)$ we set $\mathbb{N}_{\lambda}=\{(j+1,2 k-1),(j+1,2 k+1)\}$, the new grid $\mathcal{G}_{h}^{*}$ is defined as

$$
\mathcal{G}_{h}^{*}=\mathcal{G}_{j_{0}} \cup\left\{\lambda:\left|u_{\lambda}\right| \geq \varepsilon_{C}\right\} \cup_{\lambda:\left|u_{\lambda}\right| \geq \varepsilon_{R}} \mathbb{N}_{\lambda} .
$$

Using this refinement strategy we can think of a basic adaptive method where at each iteration the solution is computed and the grid is updated according to the strategy we just described. Though this is not an optimal method in terms of computational complexity, it gives us a good idea of the performance that we can expect from a more refined method in this context.

\subsection{Implementation of the method}

The implementation of the method is carried out in two stages. In the first stage, the values of the function $\phi$ as well as those of its derivatives up to order 4 are computed according at integers to the cascade algorithm described in [14]. This implies solving an eigenvector problem for a matrix of order $2 L-1, L$ being the polynomial reproduction allowed by $\phi$. By applying a refinement scheme the value of $\phi$ and of its derivatives at dyadic points in the support of $\phi$ are computed. Next we use extrapolation to compute the values of the modified functions $\varphi_{0, n}^{b}$ and $\varphi_{0}^{h b}$ at positive dyadic points, as well as the values of the modified functions $\varphi_{0, n}^{\sharp}$ and $\varphi_{0}^{h \sharp}$ at negative dyadic point of a grid at meshsize $2^{-J_{\max }}$. This stage can be performed once and for all and the values stocked.

In the second stage the actual stiffness matrix is assembled by using the stocked values. 
Table 1: Solution of trivial problem in double precision - scaling basis

\begin{tabular}{ccc}
\hline$h$ & $L^{2}$ error & $L^{\infty}$ error \\
\hline .0625 & $9.696 \mathrm{e}-06$ & $1.5677 \mathrm{e}-05$ \\
.0313 & .00015748 & .00025088 \\
.0156 & .0025464 & .0040258 \\
.0078 & .042886 & .067578 \\
.0039 & 3.0851 & 4.8962 \\
\hline
\end{tabular}

Table 2: Solution of trivial problem in double precision - wavelet basis

\begin{tabular}{ccc}
\hline$h$ & $L^{2}$ error & $L^{\infty}$ error \\
\hline .0625 & $9.696 \mathrm{e}-06$ & $1.5677 \mathrm{e}-05$ \\
.0313 & $9.8408 \mathrm{e}-06$ & $1.5677 \mathrm{e}-05$ \\
.0156 & $9.9162 \mathrm{e}-06$ & $1.5677 \mathrm{e}-05$ \\
.0078 & $9.9545 \mathrm{e}-06$ & $1.5677 \mathrm{e}-05$ \\
.0039 & $9.9734 \mathrm{e}-06$ & $1.5677 \mathrm{e}-05$ \\
\hline
\end{tabular}

In this procedure one has to take care of the precision with which the different step are computed. The high order extrapolation procedure results in an amplification of the roundoff error, which is further amplified by the multiplication by the factor $2^{4 j}$ deriving from computing the fourth derivative of the rescaled functions. This can have devastating effects on the results, as can be seen by considering the trivial problem

$$
u^{\prime \prime \prime \prime}=0, u(0)=u(1)=1, u^{\prime}(0)=u^{\prime}(1)=0
$$

whose solution is the constant function $u=1$. In order to demonstrate the issue we solved this equation on different uniform grids by performing both stages of the procedure in double precision. The results are reported in table 1.

A better (but still unsatisfactory, considering that the basis used exactly reproduces polynomials of order 11) result is obtained when using a wavelet basis (see Table 2).

The solution that we propose to this problem is to carry out the first stage of the procedure (which, we recall, is carried out once and for all), and in particular the solution of the initial small eigenvector problem and the high order extrapolation, in multiple precision. The solution of the linear system is instead carried out in double precision. In order to do it we used the Matlab Multiprecision Toolbox [28]. The results are displayed in table 4. In table 3 we present the result obtained by performing the whole computation in multiple precision, with 30 exact decimal digits.

\subsection{Numerical tests}

The Euler-Bernoulli equation describes the relationship between the beam's deflection $\omega$ and the applied load $q$. The equation reads

$$
\frac{d^{2}}{d x^{2}}\left(E(x) I(x) \frac{d^{2}}{d x^{2}} \omega(x)\right)=q(x),
$$


Table 3: Solution of trivial problem in multiple precision - 30 exact decimal digits for both stage 1 and stage 2 of the computation

\begin{tabular}{ccc}
\hline $\mathrm{h}$ & $L^{2}$ error & $L^{\infty}$ error \\
\hline .0625 & $2.3871 \mathrm{e}-25$ & $3.8769 \mathrm{e}-25$ \\
.0313 & $3.8769 \mathrm{e}-24$ & $6.1761 \mathrm{e}-24$ \\
.0156 & $6.2506 \mathrm{e}-23$ & $9.8818 \mathrm{e}-23$ \\
.0078 & $1.004 \mathrm{e}-21$ & $1.5811 \mathrm{e}-21$ \\
.0039 & $1.6095 \mathrm{e}-20$ & $2.5297 \mathrm{e}-20$ \\
\hline
\end{tabular}

Table 4: Solution of trivial problem - 30 exact decimal digits for stage 1 and double precision for stage 2

\begin{tabular}{ccc}
\hline $\mathrm{h}$ & $L^{2}$ error & $L^{\infty}$ error \\
\hline .0625 & $3.3959 \mathrm{e}-13$ & $9.952 \mathrm{e}-13$ \\
.0313 & $3.1068 \mathrm{e}-13$ & $4.6829 \mathrm{e}-13$ \\
.0156 & $6.1219 \mathrm{e}-13$ & $8.5487 \mathrm{e}-13$ \\
.0078 & $1.6684 \mathrm{e}-13$ & $5.0271 \mathrm{e}-13$ \\
.0039 & $1.0915 \mathrm{e}-13$ & $2.4181 \mathrm{e}-13$ \\
\hline
\end{tabular}

where $E$ is the elastic modulus and $I$ is the second moment of area of the beam cross section. The curve $\omega(x)$ describes the deflection of the beam in the $z$ direction at some position $x$.

\subsubsection{Test 1 .}

The first test we carried out is proposed in [30]. The equation we consider is the following:

$$
\begin{gathered}
w^{(i v)}+4 w=1, \quad \text { in }(-1,1) \\
w(-1)=w(1)=0, w^{\prime}(-1)=-w^{\prime}(1)=\frac{\sinh (2)-\sin (2)}{4(\cosh (2)+\cos (2))} .
\end{gathered}
$$

The exact solution takes the following form

$$
w=\frac{1-2(\sin (1) \sinh (1) \sin (x) \sinh (x)+\cos (1) \cosh (1) \cos (x) \cosh (x)}{4(\cosh (2)+\cos (2))}
$$

The problem is solved on a uniform grid with different mesh sizes. In table 5 we report the error, both in the $L^{2}$ and in the $L^{\infty}$ norm. In the first two columns, we report the error obtained by using multiple precision also for the solution of the linear system, while the error reported on the two columns on the right is obtained by solving the linear system in double precision. The degradation of the error as the mesh size decreases is a consequence of the ill conditioning of the matrix. This issue will of course need to be faced. However we observe on the one hand that, when solving in multiple precision, our method outperforms the sixth order method of [30], and, on the other hand, that, also when solving the linear system in double precision the results on relatively coarse grids $(h=.0625$ and $h=.0313$ ), on which 
Table 5: Results for Test 1: multiple precision (left) and double precision (right)

\begin{tabular}{lllll}
\hline $\mathrm{h}$ & $L^{2}$ error & $L^{\infty}$ error & $L^{2}$ error & $L^{\infty}$ error \\
\hline .0625 & $4.8859 \mathrm{e}-10$ & $8.071 \mathrm{e}-10$ & $4.8859 \mathrm{e}-10$ & $8.0717 \mathrm{e}-10$ \\
.0313 & $2.087 \mathrm{e}-12$ & $4.6301 \mathrm{e}-12$ & $2.8972 \mathrm{e}-12$ & $4.6153 \mathrm{e}-12$ \\
.0156 & $1.2167 \mathrm{e}-14$ & $1.9113 \mathrm{e}-14$ & $5.7798 \mathrm{e}-09$ & $2.0393 \mathrm{e}-08$ \\
.0078 & $3.2334 \mathrm{e}-17$ & $9.2613 \mathrm{e}-17$ & $4.4425 \mathrm{e}-09$ & $2.0393 \mathrm{e}-08$ \\
.0039 & $4.5559 \mathrm{e}-17$ & $9.395 \mathrm{e}-17$ & $3.1474 \mathrm{e}-08$ & $2.0393 \mathrm{e}-08$ \\
\hline
\end{tabular}
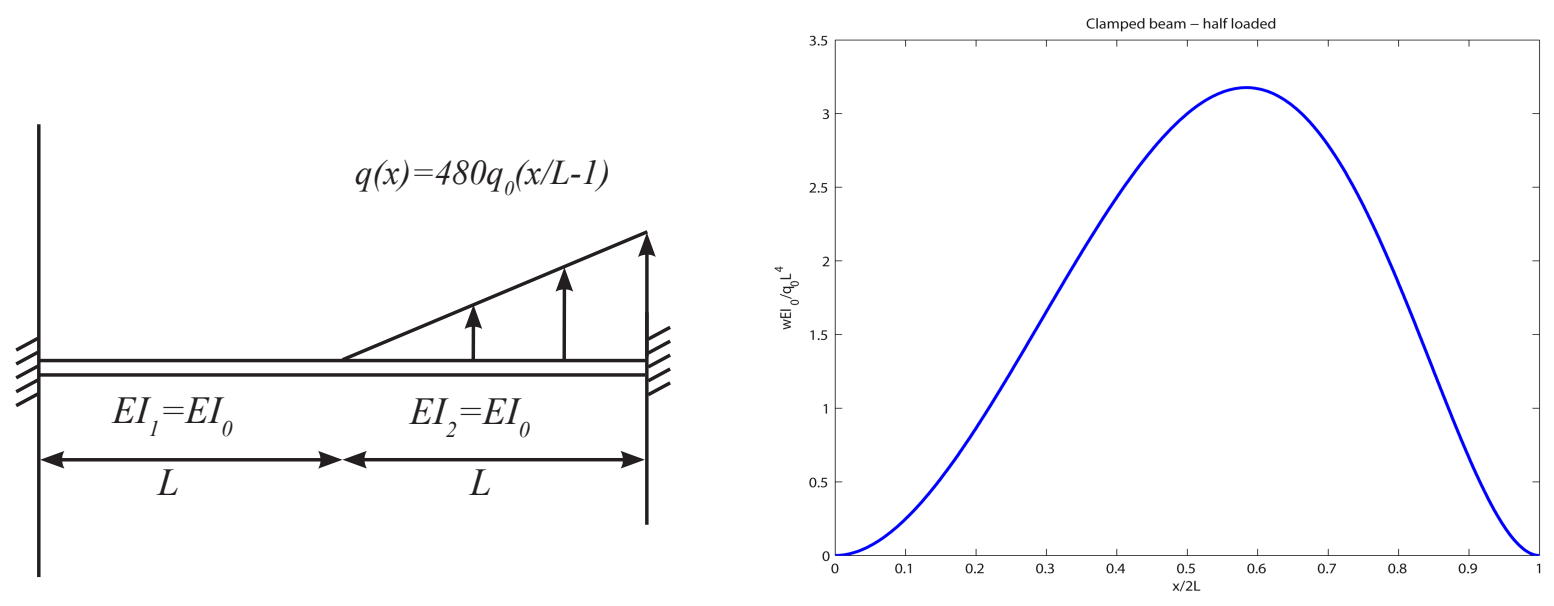

Figure 4: Clamped-clamped beam and the exact solution

the roundoff error appears to be of the same order of magnitude as the approximation error, are quite good.

\subsubsection{Test 2. Clamped-Clamped beam, half-loaded}

The second example, taken from $[15,23]$, is a non-uniformly loaded clamped beam of length $2 L$ with equal cross section (see Figure 4 ), and with constant bending rigidity $E_{0} I_{0}$. The load, limited to the right half of the beam, takes the form

$$
q(x)=\left\{\begin{array}{lll}
0 & \text { if } \quad 0 \leq x \leq L \\
k_{0} q_{0}\left(\frac{x}{L}-1\right) & \text { if } \quad L \leq x \leq 2 L
\end{array}\right.
$$

Then the exact solution of

$$
E_{0} I_{0} \frac{d^{4}}{d x^{4}} \omega(x)=q(x), \quad \omega(0)=\omega^{\prime}(0)=\omega(2 L)=\omega^{\prime}(2 L)=0
$$

is given by

$$
\omega(x)= \begin{cases}\frac{q_{0} k_{0}}{E_{0} I_{0}}\left[\frac{-L}{120} x^{3}+\frac{7 L^{2}}{480} x^{2}\right] & 0 \leq x \leq L \\ \frac{q_{0} k_{0}}{E_{0} I_{0}}\left[\frac{1}{120 L} x^{5}-\frac{1}{24} x^{4}+\frac{3 L}{40} x^{3}-\frac{11 L^{2}}{160} x^{2}+\frac{L^{3}}{24} x-\frac{L^{4}}{120}\right] & L \leq x \leq 2 L\end{cases}
$$


Table 6: Results for the clamped-clamped beam, half loaded-On the left results with uniform grids, on the left adaptive scheme

(a)

\begin{tabular}{cc}
\hline$N$ & $L^{2}$ error \\
\hline 35 & 0.0039 \\
67 & 9.8449 e-04 \\
131 & 2.4704 e-04 \\
259 & 6.1876 e-05 \\
\hline
\end{tabular}

(b)

\begin{tabular}{ccc}
\hline$\varepsilon_{R}$ & $\mathrm{~N}$ & $L^{2}$ error \\
\hline $1 . \mathrm{e}-4$ & 35 & .0039 \\
$1 . \mathrm{e}-6$ & 51 & $9.8896 \mathrm{e}-04$ \\
$1 . \mathrm{e}-8$ & 83 & $2.4859 \mathrm{e}-04$ \\
$1 . \mathrm{e}-9$ & 120 & $6.1896 \mathrm{e}-05$ \\
\hline
\end{tabular}

In the experiment, we took $k_{0}=480$, and $\frac{q_{0}}{E_{0} I_{0}}=1$ and $L=0.5$. The solution for such values of the parameters is shown on the right hand side of Figure 4.

We apply to this example the adaptive strategy described in section 3.2. In Table 6 we display, on the right hand side, the error in the $L^{2}$ norm for the result obtained with a uniform discretization for different values of the meshsize $h=2^{-J}$. On the right hand side we display the error (once again in the $L^{2}$ norm) between the true solution and the solution obtained by the adaptive strategy for different values of the tolerance $\varepsilon_{R}$ and for $\varepsilon_{C}=10^{-2} \cdot \varepsilon_{R}$. Since the solution $\omega$ is not $C^{\infty}$ but displays a jump in the 5 -th derivative, the errors for the uniform discretization are definitely bigger than the errors displayed in the previous tests (whose solution is, instead, $C^{\infty}$ ). The adaptive scheme manages to capture this lack of regularity and refines the grid at the mid-point of the segment, as shown in Figure 5. In figure 6 we show, for both uniform discretization and adaptive method, the error as a function of the number of degrees of freedom.

\section{Annexe: on Lagrange-Hermite interpolation}

The Lagrange-Hermite polynomial $\mathcal{H}_{L}$ of degree $L$, interpolating $f$ at samples $\left(x_{0}, x_{1}, \cdots, x_{L-1}\right)$ and $f^{\prime}$ at $x_{0}$ :

$$
\left\{\begin{array}{l}
\mathcal{H}_{L}\left(x_{i}\right)=f\left(x_{i}\right) \quad \forall i=0, L-1 \\
\mathcal{H}^{\prime}\left(x_{0}\right)=f^{\prime}\left(x_{0}\right),
\end{array}\right.
$$

is given by

$$
\mathcal{H}_{L}(x)=f\left(x_{0}\right) H_{0}(x)+f^{\prime}\left(x_{0}\right) H_{1}(x)+\sum_{i=1}^{L-1} f\left(x_{i}\right) L_{i}(x)
$$

where:

$$
\begin{aligned}
L_{i}(x) & =\frac{x-x_{0}}{x_{i}-x_{0}} l_{i}(x) \quad \forall i \geq 1, \\
H_{0}(x) & =\left(1-2\left(x-x_{0}\right) l_{0}^{\prime}\left(x_{0}\right)\right) l_{0}(x), \\
H_{1}(x) & =\left(x-x_{0}\right) l_{0}(x) .
\end{aligned}
$$



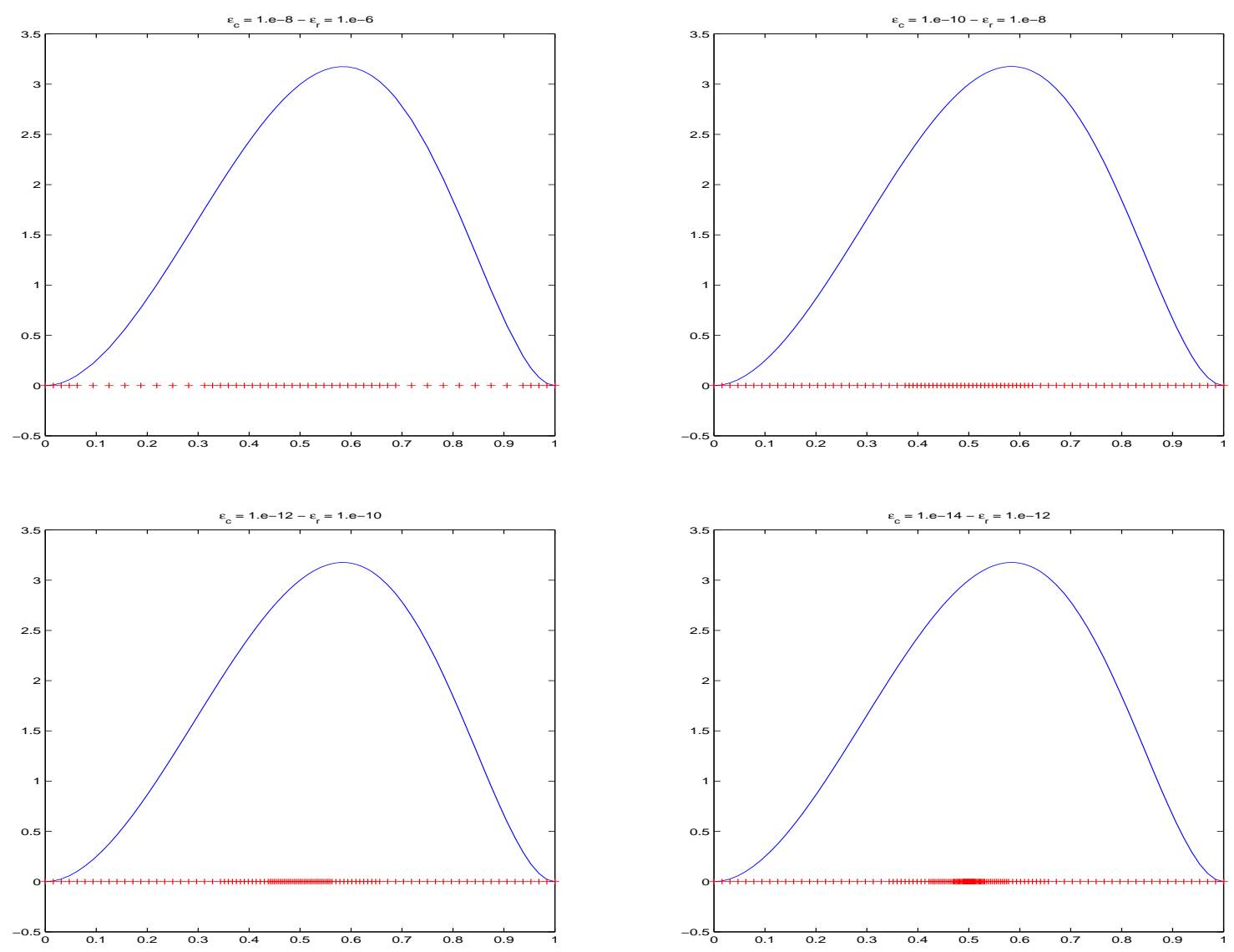

Figure 5: Clamped-clamped beam and the exact solution (in blue). Non uniform grid (in red) for values of $\varepsilon=1 . e-4,1 . e-6,1 . e-8,1 . e-9$ (from left to right and top to bottom)

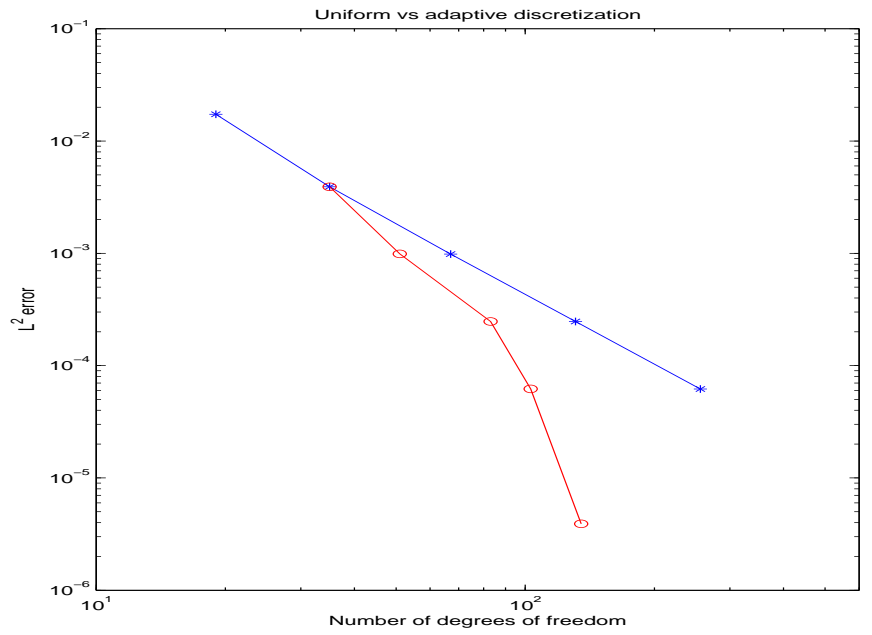

Figure 6: Error vs number of degrees of freedom for the uniform discretization (blue curve) and for the adaptive discretization (red curve) 
$l_{i}$ is the Lagrange interpolating polynomial of degree $L-1$ associated to samples $\left(x_{0}, x_{1}, \cdots, x_{L-1}\right)$ :

$$
l_{i}(x)=\prod_{q=0, q \neq i}^{L-1} \frac{x-x_{q}}{x_{i}-x_{q}}, \quad l_{0}^{\prime}\left(x_{0}\right)=\sum_{q=1}^{L-1} \frac{1}{x_{0}-x_{q}} .
$$

Example 4.1. At a given level $j>j_{1}$, for nodes of the form $i 2^{-j}$, the interpolating polynomials $L_{i}, H_{0}$ and $H_{1}$ will be denoted by $L_{j, i}, H_{j, 0}$ and $H_{j, 1}$. We separate the case $x_{0}=0$ (left), from the case $x_{0}=1$ (right) with different notations for the interpolating polynomials.

- Left extrapolation weights: at level $j$, the nodes are given by:

$$
x_{i}=i 2^{-j}, \forall i=0, \cdots, L-1 \text {. }
$$

In this case:

$$
l_{i}\left(k 2^{-j}\right)=\prod_{q=0, q \neq i}^{L-1} \frac{k-q}{i-q}, \quad l_{0}^{\prime}(0)=-\sum_{q=1}^{L-1} \frac{1}{q 2^{-j}}
$$

Then for $n \in\{1, \cdots, L-1\}$ and $\forall k \in \mathbb{Z}$,

$$
\begin{gathered}
a_{k, n}^{b}=L_{j, n}^{b}\left(k 2^{-j}\right)=\left(\frac{k}{n}\right)^{2} \prod_{q=1, q \neq i}^{L-1} \frac{k-q}{n-q} \\
a_{k, 0}^{b}=H_{j, 0}^{b}\left(k 2^{-j}\right)=\left(1+2 k \sum_{q=1}^{L-1} \frac{1}{q}\right) \prod_{q=0, q \neq i}^{L-1} \frac{k-q}{-q} \\
b_{k}^{b}=2^{j} H_{j, 1}^{b}\left(k 2^{-j}\right)=k \prod_{q=0, q \neq i}^{L-1} \frac{k-q}{-q}
\end{gathered}
$$

- Right extrapolation weights: at level $j$, the nodes are now:

and $\forall k \in \mathbb{Z}$,

$$
x_{i}=\left(2^{j}-i\right) 2^{-j}, \forall i=0, \cdots, L-1 .
$$

$$
l_{i}\left(\left(2^{j}-k\right) 2^{-j}\right)=\prod_{q=0, q \neq i}^{L-1} \frac{k-q}{i-q}, \quad l_{0}^{\prime}(1)=\sum_{q=1}^{L-1} \frac{1}{q 2^{-j}}
$$

Then $\forall n \in\{1, \cdots, L-1\}$,

$$
\begin{gathered}
a_{k, n}^{\sharp}=L_{j, n}^{\sharp}\left(\left(2^{j}-k\right) 2^{-j}\right)=\left(\frac{k}{n}\right)^{2} \prod_{q=1, q \neq i}^{L-1} \frac{k-q}{n-q} \\
a_{k, 0}^{\sharp}=H_{j, 0}^{\sharp}\left(\left(2^{j}-k\right) 2^{-j}\right)=\left(1+2 k \sum_{q=1}^{L-1} \frac{1}{q}\right) \prod_{q=0, q \neq i}^{L-1} \frac{k-q}{-q} \\
b_{k}^{\sharp}=2^{j} H_{j, 1}^{\sharp}\left(\left(2^{j}-k\right) 2^{-j}\right)=-k \prod_{q=0, q \neq i}^{L-1} \frac{k-q}{-q}
\end{gathered}
$$


Remark that, for $k=0, \cdots, L-1$, the right extrapolation weights satisfy:

$$
a_{k, n}^{\sharp}=a_{k, n}^{b}, \quad a_{k, 0}^{\sharp}=a_{k, 0}^{b}, \quad b_{k}^{\sharp}=-b_{k}^{b}
$$

and do not depend on the level $j$.

Proof of bound (23) We distinguish between $s$ integer and not. For integer $s$ we have

$$
\begin{aligned}
\langle f, g\rangle_{H^{s}(0,1)} & =\langle f, g\rangle_{H^{s-1}(0,1)}+\int_{0}^{1} f^{(s)}(x) g^{(s)}(x) d x \\
& \lesssim\|f\|_{H^{s-1}(0,1)}\|g\|_{H^{s-1}(0,1)}+\int_{0}^{1} f^{(s)}(x) g^{(s)}(x) d x
\end{aligned}
$$

We can write, for $\varepsilon<1 / 2$.

$$
\int_{0}^{1} f^{(s)}(x) g^{(s)}(x) d x \leq\left\|f^{(s)}\right\|_{H^{-\varepsilon}(0,1)}\left\|g^{(s)}\right\|_{H^{\varepsilon}(0,1)} \lesssim\|f\|_{H^{s-\varepsilon}}\|g\|_{H^{s+\varepsilon}}
$$

which, combined with the previous bound, since $s-1 \leq s-\varepsilon \leq s+\varepsilon$, yields (23).

Let us now consider $s \in(0,1)$. This time we have, for $\bar{s}=[s]$ and $\omega=s-[s]$,

$$
\begin{aligned}
\langle f, g\rangle_{H^{s}(0,1)}= & \langle f, g\rangle_{H^{\bar{s}}(0,1)}+\int_{0}^{1} d x \int_{0}^{1} d y \frac{\left(f^{(\bar{s})}(x)-f^{(\bar{s})}(y)\right)\left(g^{(\bar{s})}(x)-g^{(\bar{s})}(y)\right.}{|x-y|^{2 \omega+1}} \\
& \lesssim\|f\|_{H^{\bar{s}}(0,1)}\|g\|_{H^{\bar{s}}(0,1)}+\int_{0}^{1} d x \int_{0}^{1} d y \frac{\left(f^{(\bar{s})}(x)-f^{(\bar{s})}(y)\right)\left(g^{(\bar{s})}(x)-g^{(\bar{s})}(y)\right.}{|x-y|^{2 \omega+1}}
\end{aligned}
$$

Now, for $\varepsilon<\omega$, we can write

$$
\begin{gathered}
\int_{0}^{1} d x \int_{0}^{1} d y \frac{\left(f^{(\bar{s})}(x)-f^{(\bar{s})}(y)\right)\left(g^{(\bar{s})}(x)-g^{(\bar{s})}(y)\right.}{|x-y|^{2 \omega+1}} \\
=\int_{0}^{1} d x \int_{0}^{1} d y \frac{\left(f^{(\bar{s})}(x)-f^{(\bar{s})}(y)\right)\left(g^{(\bar{s})}(x)-g^{(\bar{s})}(y)\right.}{|x-y|^{\omega+\varepsilon+1 / 2}|x-y|^{\omega-\varepsilon+1 / 2}} \lesssim\left|f^{(\bar{s})}\right|_{H^{\omega-\varepsilon}(0,1)}\left|g^{(\bar{s})}\right|_{H^{\omega+\varepsilon}(0,1)}
\end{gathered}
$$

which, combined with the previous bound, yields the the thesis. 


\section{References}

[1] G. Beylkin, N. Saito, Wavelets, their autocorrelation functions, and multiresolution representation of signals, Intelligent Robots and Computer Vision XI: Biological, Neural Net, and 3-D Methods (D.P.Casasent, ed.), Proc. SPIE 1826, Boston, MA (1992) 39-50.

[2] S. Bertoluzza, G. Naldi, A Wavelet Collocation Method for the Numerical Solution of Partial Differential Equations, Applied and Computational Harmonic Analysis, 3, (1996) $1-9$.

[3] S. Bertoluzza, An Adaptive Collocation Method based on Interpolating Wavelets, in "Multiresolution Analysis and Wavelets for the Numerical Solution of Partial Differential Equations", W. Dahmen, A. Kurdila, P. Oswald eds., Academic Press (1997).

[4] S. Bertoluzza, Adaptive Wavelet Collocation Method for the Solution of Burgers Equation, Transport Theory and Stat. Phys., 25, (1996), 339-352.

[5] S. Bertoluzza, P. Pietra, Adaptive Wavelet Collocation for nonlinear BVPs, Proc. of ICAOS '96, Lecture Notes in Control and Information Sciences, Springer Verlag, London (1996).

[6] G. Chiavassa, J. Liandrat, On the Effective Construction of Compactly Supported Wavelets Satisfying Homogeneous Boundary Conditions on the Interval, Appl. Comput. Harmon. Anal. 4 (1997) 62-73.

[7] P.G. Ciarlet, The finite element method for elliptic problem, North Holland, Amsterdam, (1978)

[8] A. Cohen, Wavelet methods in numerical analysis, Handbook of Numerical Analysis, vol. VII, P.G. Ciarlet and J.L. Lions eds.,

[9] A. Cohen, I. Daubechies, J.-C. Feauveau, Biorthogonal bases of compactly supported wavelets, Comm. Pure Appli. Maths. 45 (1992) 485-560.

[10] A. Cohen, I. Daubechies, P. Vial, Wavelets on the Interval and Fast Wavelet Transforms, Appl. Comput. Harmon. Anal. 1 (1993) 54-81.

[11] A. Cohen, R. Masson, Wavelet Methods for Second-Order Elliptic Problems, Preconditioning, and Adaptivity. SIAM J. on Sci. Comput. 21, 1006-1026 (1999)

[12] W. Dahmen, B. Han, R.-Q. Jia, A. Kunoth, Biorthogonal Multiwavelets on the Interval: Cubic Hermite Spines, Constr. Approx., 16 (2000) 221-259.

[13] W. Dahmen, A. Kunoth, K. Urban, Biorthogonal Spline-wavelets on the interval. Stability and moment conditions, App. Comr put. Harmon. Anal. 6 (1999) 132-196.

[14] I. Daubechies, Ten Lectures on Wavelets, CBMS-NSF Regional Conference Series in Applied Mathematics, SIAM (1992). 
[15] L.A. Diaz, M.T. Martin, V. Vampa, Daubechies wavelet beam and plate finite elements, Daubechies wavelet beam and plate finite elements, Finite Elem. Anal. Des. 45 (2009) 200-209.

[16] G. Deslauriers, S. Dubuc, Symmetric Iterative Interpolation Processes, Constr. Approx. 5 (1989) 49-68.

[17] D. Donoho, Interpolating Wavelet Transform, Department of Statistics, Stanford University, preprint, 1992.

[18] A.J.M. Ferreira, L.M. Castro, S. Bertoluzza, A high order collocation method for the static and vibration analysis of composite plates using a first order theory, Composites Structures 89 (2009) 424-432.

[19] A.J.M. Ferreira, L.M. Castro, S. Bertoluzza, A wavelet collocation method for analysis of sandwich plates using a layerwise theory, Composites Structures 82 (2010) 1786-1792.

[20] A.J.M. Ferreira, L.M. Castro, S. Bertoluzza, A wavelet collocation approach for the analysis of laminated shells, Composites: Part B, 42(1) (2011).

[21] S. Grivet-Talocia, A. Tabacco, Wavelets on the interval with optimal localization, Math. Models. Meth. Appl. Sci. 10(3) (2000) 441-462.

[22] P. Joly, R. Masson, Wavelet preconditioning of the Stokes Problem in $\psi$ - $\omega$ formulation, Numerical Algorithms 24 (2000) 357-369.

[23] J. Ma, J. Xue, S. Yang, Z. He, A study of the construction and application of a Daubechies wavelet-based beam element, Finite Elem. Anal. Des. 39 (2003) 965-975.

[24] S. Mallat, A wavelet tour of signal processing, Academic Press, third edition (2009).

[25] R. Masson, Biorthogonal spline wavelets on the interval for the resolution of boundary problems. M3AS 6 (6) (1996) 749-791.

[26] P. Monasse, V. Perrier, Orthogonal Wavelet Bases Adapted For Partial Differential Equations With Boundary Conditions, SIAM J.Math. Anal. 29 (1998) 1040-1065.

[27] Y. Maday, V. Perrier, J.C. Ravel, Adaptativité dynamique sur bases d'ondelettes pour l'approximation d'équations aux dérivées partielles, C.R. Acad. Sci. Paris, Série I, 312(5), (1991) 405-410.

[28] Multiprecision Computing Toolbox for MATLAB, vX.X.X.XXXX, Advanpix LLC., Yokohama, Japan (2013).

[29] K. Schneider, O. Vasilyev, Wavelet Methods in Computational Fluid Dynamics. Ann. Rev. Fluid. Mech., (2010) 473-503.

[30] E.H. Twizell, S.I.A. Tirmizi, A sixth order method for two beam problems. Int. J. Numer. Meth. Engrg., 23 (1986) 2089-2102. 
[31] O.V. Vasilyev, N.K.-R. Kevlahan, An Adaptive Multilevel Wavelet Collocation Method for Elliptic Problems. J. Comp. Phys., 206(2) (2005) 412-431.

[32] O.V. Vasilyev, S. Paolucci, A Dynamically Adaptive Multilevel Wavelet Collocation Method for Solving Partial Differential Equations in a Finite Domain. J. Comp. Phys., 125 (1996) 498-512. 